Homology, Homotopy and Applications, vol.7(1), 2005, pp.109-150

\title{
HIGHER MONODROMY
}

\section{PIETRO POLESELLO AND INGO WASCHKIES}

\author{
(communicated by Lionel Schwartz)
}

\begin{abstract}
For a given category $C$ and a topological space $X$, the constant stack on $X$ with stalk C is the stack of locally constant sheaves with values in C. Its global objects are classified by their monodromy, a functor from the fundamental groupoid $\Pi_{1}(X)$ to $C$. In this paper we recall these notions from the point of view of higher category theory and then define the 2-monodromy of a locally constant stack with values in a 2-category $\mathbf{C}$ as a 2-functor from the homotopy 2-groupoid $\Pi_{2}(X)$ to $\mathbf{C}$. We show that 2 -monodromy classifies locally constant stacks on a reasonably well-behaved space $X$. As an application, we show how to recover from this classification the cohomological version of a classical theorem of Hopf, and we extend it to the non abelian case.
\end{abstract}

\section{Introduction}

A classical result in algebraic topology is the classification of the coverings of a (reasonably well-behaved) path-connected topological space $X$ by means of representations of its fundamental group $\pi_{1}(X)$. In the language of sheaves this generalises as an equivalence between the category of locally constant sheaves of sets on $X$ and that of representations of $\pi_{1}(X)$ on the stalk. The equivalence is given by the functor which assignes to each locally constant sheaf $\mathcal{F}$ with stalk $S$ its monodromy $\mu(\mathcal{F}): \pi_{1}(X) \rightarrow \operatorname{Aut}(S)$.

Now let $C$ be a category. It makes sense to consider monodromy representations in $\mathrm{C}$, in other words functors from the fundamental groupoid $\Pi_{1}(X)$ to $\mathrm{C}$. One would then say that they classify "locally constant sheaves on $X$ with stalk in the category C" even if there do not exist any sheaves with values in C. To state this assertion more precisely, one needs the language of stacks of Grothendieck and Giraud. A stack is, roughly speaking, a sheaf of categories and one may consider the constant stack $C_{X}$ on $X$ with stalk the category $C$ (if $C$ is the category of sets, one recovers the stack of locally constant sheaves of sets). Then one defines a local system on $X$

During the preparation of this paper, the second author was partially supported by a DAAD Post-Doc fellowship.

Received November 25, 2004, revised May 18, 2005; published on June 20, 2005.

2000 Mathematics Subject Classification: 14A20, 55P99, 18 G50.

Key words and phrases: monodromy representation, algebraic topology, stacks, category theory, non abelian cohomology.

(c) 2005, Pietro Polesello and Ingo Waschkies. Permission to copy for private use granted. 
with values in $C$ to be a global section of the constant stack $C_{X}$. The monodromy functor establishes (for a locally relatively 1-connected space $X$ ) an equivalence of categories between global sections of $C_{X}$ and functors $\Pi_{1}(X) \longrightarrow C$.

A question naturally arises: what classifies stacks on $X$ which are locally constant? Or, on the other side, which geometrical objects are classified by representations (i.e. 2-functors) of the homotopy 2-groupoid $\Pi_{2}(X)$ ? In this paper, we define a locally constant stack with values in a 2-category $\mathbf{C}$ as a global section of the constant 2-stack $\mathbf{C}_{X}$ and give an explicit construction of the 2-monodromy of such a stack as a 2-functor $\Pi_{2}(X) \longrightarrow$ C. We will show that, for locally relatively 2connected topological spaces, a locally constant stack is uniquely determined (up to equivalence) by its 2-monodromy. We then use this result to recover the cohomological version of a classical theorem of Hopf, relating the second cohomology group with constant coefficients of $X$ to its first and second homotopy group, and we extend it to the non abelian case.

During the preparation of this work, a paper [13] of B. Toen appeared, where a similar result about locally constant $\infty$-stacks and their $\infty$-monodromy is established. His approach is different from ours, since we do not use any model category theory and any simplicial techniques, but only classical 2-category (and enriched higher category) theory. Moreover, since we are only interested in the degree 2 monodromy, we need weaker hypothesis on the space $X$ than loc.cit., where the author works on the category of pointed and connected $C W$-complexes.

This paper is organised as follows. In Chapter 1 we recall some basic notions of stack theory and give a functorial construction of the classical monodromy. Our approach appears at first view to be rather heavy, as we use more language and machinery in our definition as is usually done when one considers just monodromy for sheaves of sets or abelian groups. The reason for our category theoretical approach is to motivate the construction of 2-monodromy (and to give a good idea how one could define $n$-monodromy of a locally constant $n$-stack with values in an $n$-category, for all $n$ ). As a by-product we get the classification of locally constant sheaves with values in finite categories (e.g. in the category defined by a group) which yields an amusing way to recover some non abelian versions of the "Hurewicz's formula", relating the first non abelian cohomology set with constant coefficients to representations of the fundamental group.

In Chapter 2 we introduce the 2-monodromy 2-functor of a locally constant stack with values in a 2-category. This construction is analogous to our approach to 1-monodromy, but the diagrams which should be checked for commutativity become rather large. One reason for our lengthy tale on 1-monodromy is to give good evidence to believe in our formulae, since we do not have the space to write down detailed proofs. We also describe the 2-monodromy as a descent datum on the loop space at a fixed point. Finally, we give some explicit calculations about the classification of gerbes with locally constant bands. This allows us to give a general Hurewicz-Hopf's formula, relating the second cohomology with constant coefficients (abelian or non abelian) to the representations of the fundamental groupoid of the loop space. We show how in the abelian case this reduces to the Hopf's theorem for 2-cohomology and we then give similar computations for the non abelian case. 
In Appendix A we review the definition of the stack of sheaves with values in a complete category and in Appendix B the definition of the 2-stack of stacks with values in a 2 -complete 2 -category.

Acknowledgement. We wish to thank Denis-Charles Cisinski for useful discussions and insights.

\section{Notations and conventions}

We assume that the reader is familiar with the basic notions of classical category theory, as those of category ${ }^{1}$, functor between categories, transformation between functors (also called morphism of functors), equivalence of categories, monoidal category and monoidal functor. We will also use some notions from higher category theory, as 2-categories, 2-functors, 2-transformations, modifications, 2-limits and 2 -colimits. Moreover, we will look at 3-categories, 3 -functors, etc., but only in the context of a "category enriched in 2-categories" which is much more elementary than the general theory of $n$-categories for $n \geqslant 3$. References are made to $[\mathbf{1}, \mathbf{1 1}, \mathbf{1 2}]^{2}$. For an elementary introduction to 2-limits and 2-colimits see [14].

We use the symbols C, D, etc., to denote categories. If C is a category, we denote by $\mathrm{ObC}\left(\right.$ resp. $\left.\pi_{0}(\mathrm{C})\right)$ the collection of its objects (resp. of isomorphism classes of its objects), and by $\operatorname{Hom}_{\mathrm{C}}(P, Q)$ the set of morphisms between the objects $P$ and $Q$ (if $\mathrm{C}=$ Set, the category of all small sets, we will write $\operatorname{Hom}(P, Q)$ instead of $\left.\operatorname{Hom}_{\text {Set }}(P, Q)\right)$. For a category $\mathrm{C}$, its opposite category is denoted by $\mathrm{C}^{\mathrm{op}}$.

We use the symbols $\mathbf{C}, \mathbf{D}$, etc., to denote 2-categories. If $\mathbf{C}$ is a 2-category, we denote by $\mathrm{Ob} \mathbf{C}$ (resp. $\boldsymbol{\pi}_{0}(\mathbf{C})$ ) the collection of its objects (resp. of equivalence classes of its objects), and by $\operatorname{Hom}_{\mathbf{C}}(\mathrm{P}, \mathrm{Q})$ the small category of 1-arrows between the objects $\mathrm{P}$ and $\mathrm{Q}$ (if $\mathbf{C}=\mathbf{C a t}$, the strict 2-category of all small categories, we will use the shorter notation $\operatorname{Hom}(C, D)$ to denote the category of functors between C and D). In particular, 1-arrows which are invertible up to invertible 2-arrows will be simply called equivalences. Given two 2-categories $\mathbf{C}$ and $\mathbf{D}$, the 2-category of 2 -functors from $\mathbf{C}$ to $\mathbf{D}$ will be denoted by $\operatorname{Hom}(\mathbf{C}, \mathbf{D})$. If $G$ is a commutative group, we will use the notation $\mathbf{C a t}_{G}$ for the 2-category of $G$-linear categories and $\mathrm{Hom}_{G}(\mathrm{C}, \mathrm{D})$ for the $G$-linear category of $G$-linear functors. If $\mathbf{C}$ is a 2-category, $\mathbf{C}^{\text {op }}$ denotes its opposite 2-category, meaning $\operatorname{Hom}_{\mathbf{C}_{\text {op }}}(\mathrm{P}, \mathrm{Q})=\operatorname{Hom}_{\mathbf{C}}(\mathrm{Q}, \mathrm{P})$.

\section{Locally constant sheaves with values in a category}

\subsection{Locally constant sheaves and their operations}

Let $X$ be a topological space. We denote by $\operatorname{PSt}(X)($ resp. $\mathbf{S t}(X))$ the strict 2 -category of prestacks ${ }^{3}$ (resp. stacks) of categories on $X$. For the definition of a

${ }^{1}$ There are a few well-known set-theoretical problems that arise in the definition of a category. A convenient way to overcome these difficulties has been proposed by Grothendieck using the notion of universe, and without saying so explicitly, we will work in this framework.

${ }^{2}$ Note that 2-categories are called bicategories by some authors, for whom a 2-category is what we call here a strict 2-category. A 2-functor is sometimes called a pseudo-functor.

${ }^{3}$ In this article, a prestack of categories is nothing but a contravariant 2-functor from the category of open subsets of $X$ to the 2-category Cat. This is equivalent to the notion of fibered category, 
stack on a topological space see Appendix B. The classical references are Giraud's book [9] and [SGA1, exposé VI], and a more recent one is [6]. A lighter presentation may be also found in [7].

Recall that, as for presheaves, to any prestack $\mathfrak{S}$ on $X$ one naturally associates a stack $\mathfrak{S}^{\ddagger}$. Precisely, one has the following

Proposition 1.1.1. The forgetful 2-functor

$$
\text { For : } \operatorname{St}(X) \longrightarrow \operatorname{PSt}(X)
$$

has a 2-left adjoint 2-functor

$$
\ddagger: \operatorname{PSt}(X) \longrightarrow \operatorname{St}(X) .
$$

Let us fix an adjunction 2-transformation

$$
\eta_{X}: \operatorname{Id}_{\operatorname{PSt}(X)} \longrightarrow \text { For } \circ \ddagger .
$$

Note that there is an obvious fully faithful ${ }^{4} 2$-functor of 2-categories Cat $\longrightarrow$ $\operatorname{PSt}(X)$ which associates to a category C the constant prestack $X \supset U \mapsto \mathrm{C}$.

Definition 1.1.2. Let $\mathrm{C}$ be a category. The constant stack on $X$ with stalk $\mathrm{C}$ is the image of $\mathrm{C}$ by the 2 -functor

$$
(\cdot)_{X}: \mathbf{C a t} \longrightarrow \operatorname{PSt}(X) \stackrel{\ddagger}{\longrightarrow} \operatorname{St}(X) .
$$

Note that the 2-functor $(\cdot)_{X}$ preserves faithful and fully faithful functors (hence sends (full) subcategories to (full) substacks). Moreover, the 2-transformation $\eta_{X}$ induces on global sections a natural faithful functor

$$
\eta_{X, \mathrm{C}}: \mathrm{C} \longrightarrow \mathrm{C}_{X}(X) \text {. }
$$

Definition 1.1.3. An object $\mathcal{F} \in \mathrm{ObC}_{X}(X)$ is called a local system on $X$ with values in C. A local system is constant with stalk $M$ if it is isomorphic to $\eta_{X, \mathrm{C}}(M)$ for some object $M \in \mathrm{ObC}$.

Let $\mathrm{C}=$ Set, the category of all small sets (resp. $\mathrm{C}=\operatorname{Mod}(A)$, the category of left $A$-modules for some ring $A$ ). Then it is easy to see that $C_{X}$ is naturally equivalent to the stack of locally constant sheaves of sets (resp. $A_{X}$-module). Moreover the functor $\eta_{X, \mathrm{C}}: \mathrm{C} \rightarrow \mathrm{C}_{X}(X)$ is canonically isomorphic to the functor which associates to a set $S$ (resp. an $A$-module $M$ ) the constant sheaf on $X$ with stalk $S$ (resp. $M$ ). More generally one can easily prove the following proposition:

Proposition 1.1.4. Let $\mathrm{C}$ be a complete ${ }^{5}$ category and $X$ a locally connected topological space. Denote by $\mathfrak{L}_{\mathfrak{S h}}(\mathrm{C})$ the full substack of the stack $\mathfrak{S h}_{X}(\mathrm{C})$ of sheaves with values in $\mathrm{C}$, whose objects are locally constant. Then there is a natural equivalence of stacks

$$
\mathrm{C}_{X} \stackrel{\sim}{\longrightarrow} \mathfrak{L}_{\mathfrak{c S h}}(\mathrm{C}) .
$$

and for some authors a prestack is what we call here a separated prestack (see Appendix B).

${ }^{4}$ Recall that a 2 -functor $\mathbf{F}: \mathbf{C} \rightarrow \mathbf{D}$ is faithful (resp. full, resp. fully faithful) if for any objects $\mathrm{P}, \mathrm{Q} \in \mathrm{Ob} \mathbf{C}$, the induced functor $\mathrm{F}: \operatorname{Hom}_{\mathbf{C}}(\mathrm{P}, \mathrm{Q}) \rightarrow \operatorname{Hom}_{\mathbf{D}}(\mathrm{F}(\mathrm{P}), \mathrm{F}(\mathrm{Q})$ ) is faithful (resp. full and essentially surjective, resp. an equivalence of categories).

${ }^{5}$ Recall that a complete category is a category admitting all small limits. 
For a detailed construction of the stacks $\mathfrak{S h}_{X}(\mathrm{C})$ and $\mathfrak{L} \mathfrak{c} \mathfrak{S h} \mathfrak{h}_{X}(\mathrm{C})$ see Appendix A.

Remark 1.1.5. Suppose now that $C$ is a category that is not necessarily complete. The category $\widehat{C}=\operatorname{Hom}\left(\mathrm{C}^{\mathrm{op}}\right.$, Set) is complete (and cocomplete) and the Yoneda embedding

$$
Y: \mathrm{C} \longrightarrow \widehat{\mathrm{C}}, \quad P \mapsto \operatorname{Hom}_{\mathrm{C}}(\cdot, P)
$$

commutes to small limits. Then one usually defines sheaves with values in $C$ as presheaves that are sheaves in the category $\mathrm{PSh}_{X}(\widehat{\mathrm{C}})$. Note that in general there does not exist a sheaf with values in $C$ (take for example $C$ the category of finite sets), but if $\mathrm{C} \neq \varnothing$ then the category $\mathrm{C}_{X}(X)$ of local systems with values in $\mathrm{C}$ is always non-empty. Still, even in that case, we will sometimes refer to a local system as a locally constant sheaf. More precisely we get a fully faithful functor of stacks

$$
Y_{X}: \mathrm{C}_{X} \longrightarrow(\widehat{\mathrm{C}})_{X} \simeq \mathfrak{L} \mathfrak{c} \mathfrak{S} \mathfrak{h}_{X}(\widehat{\mathrm{C}})
$$

Let $\mathcal{F}$ be an object of $\mathfrak{L} \mathfrak{c} \mathfrak{S} \mathfrak{h}_{X}(\widehat{\mathcal{C}})$ on some open set. Then $\mathcal{F}$ is in the essential image of $Y_{X}$ if and only if all of its stalks are representable.

Let $f: X \rightarrow Y$ be a continuous map of topological spaces, $\mathfrak{S}$ a prestack on $X$ and $\mathfrak{D}$ a prestack on $Y$.

Notation 1.1.6. (i) Denote by $f_{*} \mathfrak{S}$ the prestack on $Y$ such that, for any open set $V \subset Y, f_{*} \mathfrak{S}(V)=\mathfrak{S}\left(f^{-1}(V)\right)$. If $\mathfrak{S}$ is a stack on $X$, then $f_{*} \mathfrak{S}$ is a stack on $Y$.

(ii) Denote by $f_{p}^{-1} \mathfrak{D}$ the prestack on $X$ such that, for any open set $U \subset X$, $f_{p}^{-1} \mathfrak{D}(U)=\underset{f(\overrightarrow{U) \subset V}}{2 \lim } \mathfrak{D}(V)$. If $\mathfrak{D}$ is a stack on $Y$, we set $f^{-1} \mathfrak{D}=\left(f_{p}^{-1} \mathfrak{D}\right)^{\ddagger}$.

Recall that the category $2 \underset{\longrightarrow}{2} \mathfrak{D}(V)$ is described as follows:

$$
f(\overrightarrow{U) \subset V}
$$

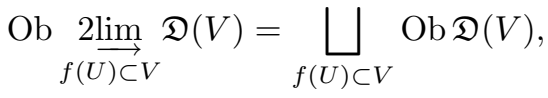

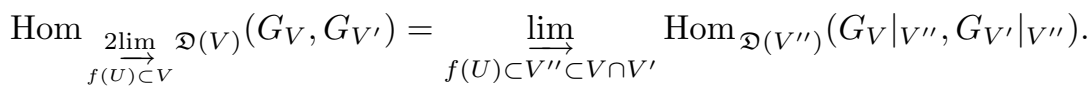

Proposition 1.1.7. The 2-functors

$$
f_{*}: \mathbf{S t}(X) \longrightarrow \mathbf{S t}(Y) \quad f^{-1}: \mathbf{S t}(Y) \longrightarrow \mathbf{S t}(X)
$$

are 2-adjoint, $f_{*}$ being the right 2-adjoint of $f^{-1}$.

Moreover, if $g: Y \rightarrow Z$ is another continuous map, one has natural equivalences ${ }^{6}$ of 2-functors

$$
g_{*} \circ f_{*} \simeq(g \circ f)_{*}, \quad f^{-1} \circ g^{-1} \simeq(g \circ f)^{-1} .
$$

${ }^{6}$ For sake of simplicity, here and in the sequel we use the word "equivalence" for a 2-transformation which is invertible up to an invertible modification. In the case of the inverse image, to be natural means that if we consider the continuous maps $h=f_{3} \circ f_{2} \circ f_{1}, g_{1}=f_{2} \circ f_{1}$ and $g_{2}=f_{3} \circ f_{2}$, then the two equivalences $h^{-1} \simeq f_{1}^{-1} g_{2}^{-1} \simeq f_{1}^{-1} f_{2}^{-1} f_{3}^{-1}$ and $h^{-1} \simeq g_{1}^{-1} f_{3}^{-1} \simeq f_{1}^{-1} f_{2}^{-1} f_{3}^{-1}$ are naturally isomorphic by a modification, in the sense that, if we look at the continuous map $k=f_{4} \circ f_{3} \circ f_{2} \circ f_{1}$, we get the obvious commutative diagram of modifications. 
Since the following diagram commutes up to equivalence

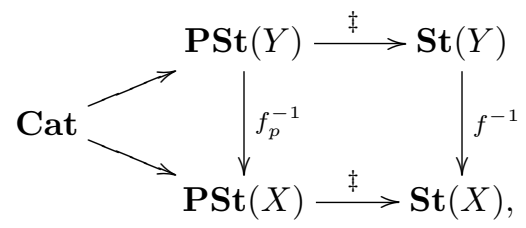

the 2 -functor $f^{-1}$ preserves constant stacks (up to natural equivalence).

Definition 1.1.8. Denote by $\Gamma(X, \cdot)$ the 2 -functor of global sections

$$
\operatorname{St}(X) \longrightarrow \text { Cat, } \quad \mathfrak{S} \mapsto \Gamma(X, \mathfrak{S})=\mathfrak{S}(X)
$$

and set $\Gamma_{X}=\Gamma(X, \cdot) \circ(\cdot)_{X}$.

Note that for any stack $\mathfrak{S}$, the category $\mathfrak{S}(\varnothing)$ is the terminal object in Cat (which consists of precisely one morphism). Hence the 2 -functor

$$
\Gamma(\{\mathrm{pt}\}, \cdot): \operatorname{St}(\{\mathrm{pt}\}) \longrightarrow \mathbf{C a t}
$$

is an equivalence of 2-categories. We easily deduce

Proposition 1.1.9. The 2-functor $\Gamma(X, \cdot)$ is right 2-adjoint to $(\cdot)_{X}$.

It is not hard to see that we may choose the functors $\eta_{X, \mathrm{C}}: \mathrm{C} \longrightarrow \Gamma\left(X, \mathrm{C}_{X}\right)$ to define the adjunction 2-transformation

$$
\eta_{X}: \mathrm{Id}_{\mathrm{Cat}} \longrightarrow \Gamma_{X}
$$

Consider the commutative diagram of topological spaces

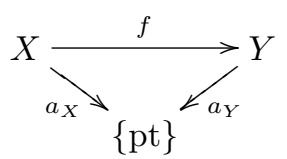

and the induced 2-transformation of 2-functors

$$
a_{Y *} \circ(\cdot)_{Y} \longrightarrow a_{Y *} \circ f_{*} \circ f^{-1} \circ(\cdot)_{Y} \simeq a_{X *} \circ f^{-1} \circ(\cdot)_{Y} \simeq a_{X *} \circ(\cdot)_{X} .
$$

Hence we get a 2-transformation of 2-functors $f^{-1}$ compatible with $\eta_{X}$ and $\eta_{Y}$, i.e. such that the following diagram commutes up to a natural invertible modification:

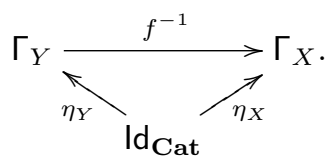

Note that this implies that for each point $x \in X$ and any local systems $\mathcal{F}, \mathcal{G} \in$ $\mathrm{C}_{X}(X)$, the natural morphism

$$
i_{x}^{-1} \mathcal{H o m}_{\mathrm{C}_{\mathrm{X}}}(\mathcal{F}, \mathcal{G}) \rightarrow \operatorname{Hom}_{\mathrm{C}}\left(i_{x}^{-1} \mathcal{F}, i_{x}^{-1} \mathcal{G}\right)
$$


is an isomorphism (here $i_{x}:\{\mathrm{pt}\} \rightarrow X$ denotes the natural map sending $\{\mathrm{pt}\}$ to $x$ and we identify $C$ with global sections of $C_{p t}$ ). Therefore, for any continuous map $f: X \rightarrow Y$, we get a natural isomorphism of locally constant sheaves of sets

$$
f^{-1} \mathcal{H} \text { om }_{\mathrm{C}_{Y}}(\mathcal{F}, \mathcal{G}) \stackrel{\sim}{\longrightarrow} \mathcal{H}_{\text {om }} \mathrm{C}_{X}\left(f^{-1} \mathcal{F}, f^{-1} \mathcal{G}\right) .
$$

Now we are ready to formulate the fundamental Lemma of homotopy invariance of local systems as follows:

Lemma 1.1.10. Let $I=[0,1]$ and $t \in I$. Consider the maps

$$
X \underset{p}{\stackrel{\iota_{t}}{\rightleftarrows}} X \times I
$$

where $\iota_{t}(x)=(x, t)$ and $p$ is the projection. Then the 2-transformations

$$
\Gamma_{X} \underset{\iota_{t}^{-1}}{\stackrel{p^{-1}}{<}} \Gamma_{X \times I}
$$

are equivalences of 2-functors, quasi-inverse one to each other.

Proof. Let $\mathrm{C}$ be a category. It is sufficient to show that the functors

$$
\Gamma\left(X, \mathrm{C}_{X}\right) \underset{\iota_{t}^{-1}}{\stackrel{p^{-1}}{\gtrless}} \Gamma\left(X \times I, \mathrm{C}_{X \times I}\right)
$$

are natural equivalences of categories, quasi-inverse one to each other. Since $\iota_{t}^{-1}$ 。 $p^{-1} \simeq\left(p \circ \iota_{t}\right)^{-1}=\mathrm{id}_{\Gamma\left(X, \mathrm{C}_{X}\right)}$, it remains to check that for each $\mathcal{F} \in \Gamma\left(X \times I, \mathrm{C}_{X \times I}\right)$ there is a natural isomorphism $p^{-1} \iota_{t}^{-1} \mathcal{F} \simeq \mathcal{F}$.

First let us prove that if $\mathcal{F}$ is a locally constant sheaf of sets on $X \times I$, then the natural morphism

$$
\iota_{t}^{-1}: \Gamma(X \times I, \mathcal{F}) \rightarrow \Gamma\left(X, \iota_{t}^{-1} \mathcal{F}\right)
$$

is an isomorphism. Indeed, let $s$ and $s^{\prime}$ be two sections in $\Gamma(X \times I, \mathcal{F})$ such that $s_{x, t}=s_{x, t}^{\prime}$. Since $\mathcal{F}$ is locally constant, the set $\left\{t^{\prime} \in I \mid s_{x, t^{\prime}}=s_{x, t}\right\}$ is open and closed, hence equal to $I$. Therefore the map is injective. Now let $s \in \Gamma\left(X, i_{t}^{-1} \mathcal{F}\right)$. Then $s$ is given by sections $s_{j}$ of $\mathcal{F}$ on a family $\left(U_{j} \times I_{j}\right)_{j \in J}$ where $I_{j}$ is an open interval containing $t$, the $U_{j}$ cover $X$ and the sheaf $\mathcal{F}$ is constant on $U_{j} \times I_{j}$. It is not hard to see that, by refining the covering, the sections $s_{j}$ can be extended to $U_{j} \times I$ and using the injectivity of the map one sees that we can patch the extensions of the $s_{j}$ to get a section of $\mathcal{F}$ on $X \times I$ that is mapped to $s$. Hence the morphism (1.1.2) is an isomorphism.

Now let $\mathcal{F} \in \Gamma\left(X \times I, C_{X \times I}\right)$. Since $\iota_{t}^{-1} \mathcal{H} \operatorname{Com}_{\mathrm{C}_{X \times I}}\left(p^{-1} \iota_{t}^{-1} \mathcal{F}, \mathcal{F}\right) \stackrel{\sim}{\longrightarrow} \mathcal{H} m_{\mathrm{C}_{X}}\left(\iota_{t}^{-1} \mathcal{F}\right.$, $\left.\iota_{t}^{-1} \mathcal{F}\right)$, we get the isomorphism

$$
\Gamma\left(X \times I, \mathcal{H}{ }^{\prime} m_{\mathrm{C}_{X \times I}}\left(p^{-1} \iota_{t}^{-1} \mathcal{F}, \mathcal{F}\right)\right) \stackrel{\sim}{\longrightarrow} \Gamma\left(X, \mathcal{H} \operatorname{lom}_{\mathrm{C}_{X}}\left(\iota_{t}^{-1} \mathcal{F}, \iota_{t}^{-1} \mathcal{F}\right)\right) .
$$

It is an easy exercise to verify that we can define the isomorphism $p^{-1} \iota_{t}^{-1} \mathcal{F} \stackrel{\sim}{\longrightarrow} \mathcal{F}$ by the identity section in the set $\Gamma\left(X, \mathcal{H} \operatorname{Fom}_{\mathrm{C}_{X}}\left(\iota_{t}^{-1} \mathcal{F}, \iota_{t}^{-1} \mathcal{F}\right)\right)$. 
Taking $X=\{\mathrm{pt}\}$ in Lemma 1.1.10, we get

Corollary 1.1.11. The adjunction 2-transformation

$$
\eta_{I}: \mathrm{Id}_{\mathbf{C a t}} \longrightarrow \Gamma_{I}
$$

is an equivalence, i.e. for each category $\mathrm{C}$ the functor $\eta_{I, \mathrm{C}}: \mathrm{C} \longrightarrow \Gamma\left(I, \mathrm{C}_{I}\right)$ is a natural equivalence.

For each $X$ and each $t \in I$, we have an invertible modification $\operatorname{id}_{\Gamma_{X}} \simeq \iota_{t}^{-1} p^{-1}$. Therefore, for any $s, t \in I$ there exists a canonical invertible modification $\iota_{t}^{-1} \simeq \iota_{s}^{-1}$ which can be used to prove the following technical Lemma:

Lemma 1.1.12. The diagram of continuous maps on the left induces for any $s, t, t^{\prime} \in I$ the commutative diagram of modifications on the right
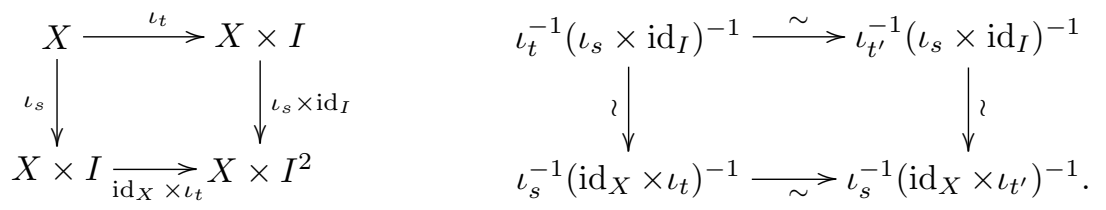

Moreover, let $H: X \times I \rightarrow Y$ be a continuous map that factors through the projection $p: X \times I \rightarrow X$. Then the composition of invertible modifications

$$
\left(H \circ \iota_{t}\right)^{-1} \simeq \iota_{t}^{-1} H^{-1} \simeq \iota_{t^{\prime}}^{-1} H^{-1} \simeq\left(H \circ \iota_{t^{\prime}}\right)^{-1}
$$

is the identity.

Let Top denote the strict 2-category of topological spaces and continuous maps, where 2-arrows are homotopy classes of homotopies between functions (see for example [1, Chapter 7] for explicit details). Then homotopy invariance of locally constant sheaves may be expressed as the following

Proposition 1.1.13. The assignment $(\mathrm{C}, X) \mapsto \Gamma\left(X, \mathrm{C}_{X}\right)$ defines a 2-functor

$$
\Gamma: \text { Cat } \times \text { Top }^{\text {op }} \longrightarrow \text { Cat. }
$$

Moreover, the natural functors $\eta_{X, \mathrm{C}}: \mathrm{C} \longrightarrow \Gamma(X, \mathrm{C})$ define a 2-transformation

$$
\eta: \mathrm{Q}_{1} \longrightarrow \Gamma
$$

where $\mathrm{Q}_{1}:$ Cat $\times$ Top ${ }^{\mathrm{op}} \longrightarrow$ Cat is the projection .

Proof. It remains to check that $\Gamma$ is well defined at the level of 2 -arrows. Let $f_{i}: X \rightarrow$ $Y$ be continuous maps for $i=0,1$ and let $H: f_{0} \rightarrow f_{1}$ be a homotopy, that is, a continuous map $X \times I \rightarrow Y$ such that $H \circ \iota_{0}=f_{0}$ and $H \circ \iota_{1}=f_{1}$. Then $\alpha_{H}: f_{0}^{-1} \stackrel{\sim}{\longrightarrow} f_{1}^{-1}$ is defined by the chain of natural invertible modifications

$$
f_{0}^{-1}=\left(H \circ \iota_{0}\right)^{-1} \simeq \iota_{0}^{-1} H^{-1} \simeq \iota_{1}^{-1} H^{-1} \simeq\left(H \circ \iota_{1}\right)^{-1}=f_{1}^{-1} \text {. }
$$

Consider the constant homotopy at $f: X \rightarrow Y$

$$
H_{f}: X \times I \rightarrow Y, \quad(x, t) \mapsto f(x) .
$$


Since we may factor $H_{f}$ as $X \times I \stackrel{p}{\rightarrow} X \stackrel{f}{\rightarrow} Y$, by Lemma 1.1 .12 we get $\alpha_{H_{f}}=\mathrm{id}_{\mathrm{f}-1}$. Now let $H_{0}, H_{1}: f_{0} \rightarrow f_{1}$ be two homotopies and $K: H_{0} \rightarrow H_{1}$ a homotopy. Then consider the commutative diagram of invertible modifications

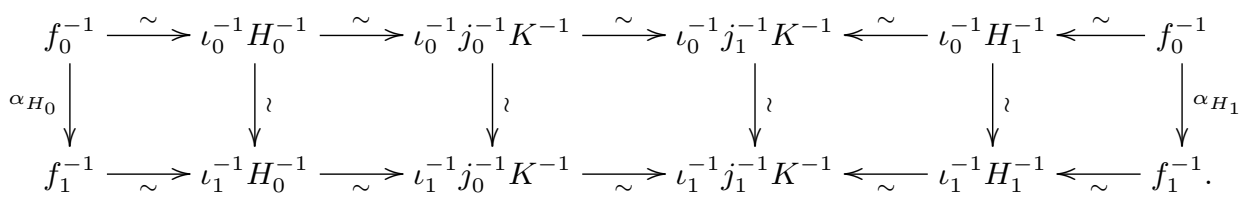

We have to check that the horizontal lines are identity modifications. This is a consequence of Lemma 1.1.12, which allows us (by a diagram chase) to identify the two lines with the modifications induced by constant homotopies.

The fact that $\alpha$ is compatible with the composition of homotopies is finally a very easy diagram chase.

\subsection{The monodromy functor}

Definition 1.2.1. The homotopy groupoid (or fundamental groupoid) of $X$ is the small groupoid $\Pi_{1}(X)=\operatorname{Hom}_{\text {Top }}(\{\mathrm{pt}\}, X)$.

Roughly speaking, objects of $\Pi_{1}(X)$ are the points of $X$ and for any $x, y \in X$, $\operatorname{Hom}_{\Pi_{1}(X)}(x, y)$ is the set of homotopy classes of paths starting from $x$ and ending at $y$. The composition law is the opposite of the composition of paths. Note that, in particular, $\pi_{0}\left(\Pi_{1}(X)\right)=\pi_{0}(X)$, the set of arcwise connected components of $X$, and for each $x \in X, \operatorname{Hom}_{\Pi_{1}(X)}(x, x)=\pi_{1}(X, x)$, the fundamental group of $X$ at $x$.

Let $\mathbf{G r}$ denote the strict 2 -category of small groupoids. Then we have a 2 -functor

$$
\Pi_{1}: \text { Top } \longrightarrow \mathbf{G r}
$$

which defines the Yoneda type 2-functor

$$
\mathrm{Y}_{\Pi_{1}}: \text { Cat } \times \text { Top }^{\text {op }} \longrightarrow \text { Cat }, \quad(\mathrm{C}, X) \mapsto \operatorname{Hom}\left(\Pi_{1}(X), \mathrm{C}\right) .
$$

Definition 1.2.2. The monodromy 2-transformation

$$
\mu:\left\ulcorner\longrightarrow \mathrm{Y}_{\Pi_{1}}\right.
$$

is defined as follows. For any topological space $X$ and any category C, the 2-functor $\Gamma$ defines a functor

$$
\operatorname{Hom}_{\text {Top }}(\{\mathrm{pt}\}, X) \longrightarrow \operatorname{Hom}\left(\Gamma\left(X, \mathrm{C}_{X}\right), \mathrm{C}\right),
$$

which induces by evaluation a natural functor

$$
\Gamma\left(X, \mathrm{C}_{X}\right) \times \Pi_{1}(X) \longrightarrow \mathrm{C}
$$

and hence, by adjunction, a functor

$$
\mu_{X, \mathrm{C}}: \Gamma\left(X, \mathrm{C}_{X}\right) \longrightarrow \operatorname{Hom}\left(\Pi_{1}(X), \mathrm{C}\right) .
$$

We will sometimes use the shorter notation $\mu$ instead of the more cumbersome $\mu_{X, \mathrm{C}}$. We will also extend $\mu$ to pointed spaces without changing the notations. 
Let us briefly illustrate that we have constructed the well-known classical monodromy functor. Recall that, for each $x \in X$ one has a natural stalk 2-functor

$$
\mathrm{F}_{x}: \operatorname{St}(X) \longrightarrow \text { Cat, } \quad \mathfrak{S} \mapsto \mathfrak{S}_{x}=2 \lim _{x \in U} \mathfrak{S}(U) .
$$

Let $i_{x}:\{x\} \rightarrow X$ denote the natural embedding. Since $\mathrm{F}_{x}$ is canonically equivalent to $\Gamma(\{x\}, \cdot) \circ i_{x}^{-1}$, one gets a 2-transformation $\Gamma(X, \cdot) \rightarrow \mathrm{F}_{x}$ and then a 2transformation

$$
\rho_{x}: \Gamma_{X} \longrightarrow \mathrm{F}_{X, x}=\mathrm{F}_{x} \circ(\cdot)_{X} .
$$

For an object $\mathcal{F}$ (resp. morphism $f$ ) in $C_{X}(X)$, if there is no risk of confusion, we will simply write $\mathcal{F}_{x}$ (resp. $f_{x}$ ) to denote $\rho_{x, \mathrm{C}}(\mathcal{F})$ (resp. $\left.\rho_{x, \mathrm{C}}(f)\right)$ in $\left(\mathrm{C}_{X}\right)_{x}$.

Let Top $_{*}$ be the strict 2-category of pointed topological spaces, pointed continuous maps and homotopy classes of pointed homotopies. One can prove by diagram chases similar to those in the proof of Proposition 1.1.13, that

Proposition 1.2.3. The assignment $(\mathrm{C},(X, x)) \mapsto\left(\mathrm{C}_{X}\right)_{x}$ defines a 2-functor

$$
\text { F: Cat } \times \text { Top }_{*}^{\text {op }} \longrightarrow \text { Cat. }
$$

Moreover, the natural functors $\rho_{x, \mathrm{C}}: \Gamma\left(X, \mathrm{C}_{X}\right) \longrightarrow\left(\mathrm{C}_{X}\right)_{x}$ define a 2-transformation

$$
\rho: \Gamma \longrightarrow \mathrm{F} \text {. }
$$

Since the stalks of the stack associated to a prestack do not change, for each category $\mathrm{C}$ and each pointed space $(X, x)$ the functor

$$
\mathrm{C} \stackrel{\eta_{X, \mathrm{c}}}{\longrightarrow} \mathrm{C}_{X}(X) \stackrel{\rho_{x, \mathrm{c}}}{\longrightarrow}\left(\mathrm{C}_{X}\right)_{x}
$$

is an equivalence of categories. Hence the composition

$$
\rho \circ \eta: \mathrm{Q}_{1} \longrightarrow \mathrm{F}
$$

is an equivalence of 2-functors (here $\mathrm{Q}_{1}$ : Cat $\times \mathbf{T o p}_{*}^{\mathrm{op}} \longrightarrow$ Cat is the natural projection). Let $\varepsilon: \mathrm{F} \longrightarrow \mathrm{Q}_{1}$ denote a fixed quasi-inverse to $\rho \circ \eta$, i.e. for each category $\mathrm{C}$ and each pointed space $(X, x)$, we fix a natural equivalence

$$
\varepsilon_{x, \mathrm{C}}:\left(\mathrm{C}_{X}\right)_{x} \stackrel{\sim}{\longrightarrow} \mathrm{C}
$$

such that if we have a pointed continuous map $f:(X, x) \rightarrow(Y, y)$ we get a diagram

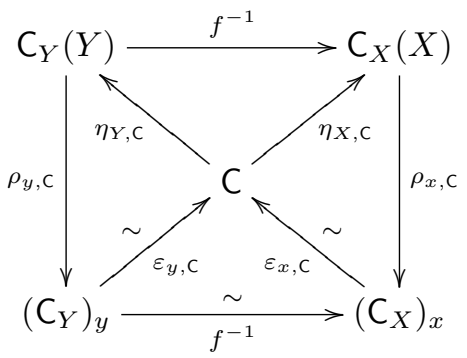

that commutes up to natural isomorphism. Denote by $\omega$ the composition $\varepsilon \circ \rho: \Gamma \longrightarrow$ $\mathrm{Q}_{1}$. Fix a topological space $X$ and a category $\mathrm{C}$, and let $\mathcal{F} \in \Gamma\left(X, \mathrm{C}_{X}\right)$. Then a direct 
comparison shows that, up to natural isomorphism, we have

$$
\mu_{X, \mathrm{C}}(\mathcal{F})(x)=\omega_{x, \mathrm{C}}(\mathcal{F})
$$

(if $\mathrm{C}=$ Set, then $\omega_{x}$ is just the usual stalk-functor) and if $\gamma: x \rightarrow y$ is a path, then $\mu_{X, C}(\mathcal{F})(\gamma)$ is defined by the chain of isomorphisms

$$
\omega_{x, \mathrm{C}}(\mathcal{F}) \simeq \omega_{0, \mathrm{C}}\left(\gamma^{-1} \mathcal{F}\right) \simeq \eta_{I, \mathrm{C}}^{-1}\left(\gamma^{-1} \mathcal{F}\right) \simeq \omega_{1, \mathrm{C}}\left(\gamma^{-1} \mathcal{F}\right) \simeq \omega_{y, \mathrm{C}}(\mathcal{F})
$$

(and if $\mathrm{C}=$ Set, we usually choose $\eta_{I}^{-1}=\Gamma(I, \cdot)$ ).

In particular, this means that the following diagram commutes up to natural invertible modification

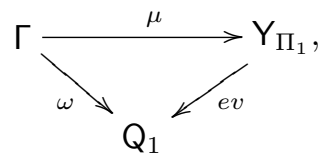

where $e v$ is the evaluation 2-transformation, that is for each pointed space $(X, x)$ and each functor $\alpha: \Pi_{1}(X) \rightarrow \mathrm{C}$, it is defined by $e v_{x}(\alpha)=\alpha(x)$.

Let $\Delta: \mathrm{Q}_{1} \longrightarrow \mathrm{Y}_{\Pi_{1}}$ denote the diagonal 2-transformation: for each topological space $X$, each category $\mathrm{C}$ and each $M \in \mathrm{ObC}, \Delta_{X, \mathrm{C}}(M)$ is the constant functor $x \mapsto M$ (i.e. the trivial representation with stalk $M$ ). Clearly $e v \circ \Delta=\mathrm{id}_{\mathrm{Q}_{1}}$. Moreover, by a diagram chase, we easily get

Proposition 1.2.4. The diagram of 2-transformations

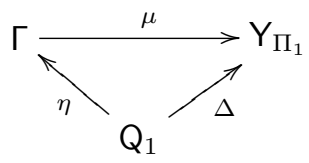

commutes up to invertible modification.

Proposition 1.2.5. For each topological space $X$ and each category $\mathrm{C}$, the functor

$$
\mu_{X, \mathrm{C}}: \Gamma\left(X, \mathrm{C}_{X}\right) \longrightarrow \operatorname{Hom}\left(\Pi_{1}(X), \mathrm{C}\right) .
$$

is faithful and conservative.

Proof. Let $f, g: \mathcal{F} \rightarrow \mathcal{G}$ be two morphisms of local systems such that $\mu(f)=\mu(g)$. Since the diagram (1.2.1) commutes and $\varepsilon_{x, \mathrm{C}}:\left(\mathrm{C}_{X}\right)_{x} \longrightarrow \mathrm{C}$ is an equivalence, we get that $f_{x}=g_{x}$ in $\left(\mathrm{C}_{X}\right)_{x}$ for all $x \in X$. Since $\mathrm{C}_{X}$ is a stack, this implies that $f=g$, hence $\mu_{X, C}$ is faithful.

The same diagram implies that if $\mu(f)$ is an isomorphism, then $f_{x}$ is an isomorphism in $\left(C_{X}\right)_{x}$ for all $x \in X$ and therefore $f$ is an isomorphism.

Proposition 1.2.6. Let $X$ be locally arcwise connected. Then for each category C, the functor

$$
\mu_{X, \mathrm{C}}: \Gamma\left(X, \mathrm{C}_{X}\right) \longrightarrow \operatorname{Hom}\left(\Pi_{1}(X), \mathrm{C}\right)
$$

is full. 
Proof. Let $\mathcal{F}, \mathcal{G} \in \mathrm{C}_{X}(X)$. A morphism $\phi: \mu(\mathcal{F}) \rightarrow \mu(\mathcal{G})$ is given by a family of morphisms

$$
\phi_{x}: e v_{x}(\mu(\mathcal{F})) \rightarrow e v_{x}(\mu(\mathcal{G}))
$$

such that for every (homotopy class of) path $\gamma: x \rightarrow y$, the diagram

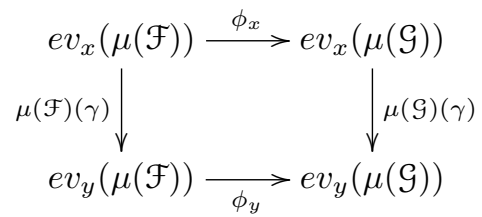

is commutative. Using the diagram (1.2.1) and the definition of the stalk of a stack, we get an arcwise connected open neighbourhood $U_{x}$ of $x$ and a morphism

$$
\varphi^{x}:\left.\left.\mathcal{F}\right|_{U_{x}} \rightarrow \mathcal{G}\right|_{U_{x}}
$$

such that $e v_{x}\left(\mu\left(\varphi^{x}\right)\right)=\phi_{x}$.

In order to patch the $\varphi^{x}$, we have to show that for any $z \in U_{x} \cap U_{y}$ we have $\left(\varphi^{x}\right)_{z}=\left(\varphi^{y}\right)_{z}$. Since $\varepsilon_{z}$ is an equivalence, it is sufficient to check that $e v_{z}\left(\mu\left(\varphi^{x}\right)\right)=$ $e v_{z}\left(\mu\left(\varphi^{y}\right)\right)$.

Choose a path $\gamma: x \rightarrow z$. Then

$$
\mu(\mathcal{G})(\gamma) \circ e v_{x}\left(\mu\left(\varphi^{x}\right)\right) \circ \mu(\mathcal{F})(\gamma)^{-1}=e v_{z}\left(\mu\left(\varphi^{x}\right)\right) .
$$

But by definition the lefthand side is just $\phi_{z}$. Similarly, taking a path $\gamma^{\prime}: y \rightarrow z$, we get

$$
e v_{z}\left(\mu\left(\varphi^{x}\right)\right)=\phi_{z}=e v_{z}\left(\mu\left(\varphi^{y}\right)\right)
$$

and, by definition of the stalk, this means that $\varphi^{x}$ and $\varphi^{y}$ coincide in a neighbourhood of $z$. Since $z$ was chosen arbitrarily, they coincide on $U_{x} \cap U_{y}$. Since $C_{X}$ is a stack, we can patch the morphisms $\varphi_{x}$ to a unique $\varphi: \mathcal{F} \rightarrow \mathcal{G}$ such that $\mu(\varphi)=\phi$.

Corollary 1.2.7. Let $X$ be 1-connected ${ }^{7}$. Then

$$
\eta_{X}: \operatorname{ld}_{\text {Cat }} \longrightarrow \Gamma_{X}
$$

is an equivalence of 2-functors, i.e. for each category $\mathrm{C}$, the functor $\eta_{X, \mathrm{C}}: \mathrm{C} \longrightarrow$ $\Gamma\left(X, C_{X}\right)$ is an equivalence of categories, natural in $\mathrm{C}$.

Proof. Fix $x_{0} \in X$. Since the groupoid $\Pi_{1}(X)$ is trivial (that is, $\Pi_{1}(X)$ reduces to the category with a single object and only the identity morphism), the 2-transformation $e v_{x_{0}}: \mathbf{Y}_{\Pi_{1}(X)} \longrightarrow \mathrm{Id}_{\text {Cat }}$ is an equivalence, quasi-inverse to $\mu_{X} \circ \eta_{X}$. Since $\mu_{X}$ is fully faithful, this implies by abstract nonsense that $\mu_{X}$ and $\eta_{X}$ are equivalences.

Denote by $\mathbf{C S t}(X)$ the full 2-subcategory of $\mathbf{S t}(X)$ of constant stacks on $X$. We get

${ }^{7}$ Here and for the sequel, a topological space $X$ is $n$-connected if $\pi_{i}(X) \simeq 1$ for all $0 \leqslant i \leqslant n$, and locally $n$-connected if each neighbourhood of each point contains an $n$-connected neighbourhood. 
Corollary 1.2.8. If $X$ is 1-connected, the functors

$$
\text { Cat } \underset{\Gamma(X, \cdot)}{\stackrel{(\cdot)_{X}}{\rightleftarrows}} \operatorname{CSt}(X)
$$

are equivalences of 2-categories, inverse one to each other.

Theorem 1.2.9. Let $X$ be locally relatively 1 -connected ${ }^{8}$. Then the monodromy

$$
\mu_{X}: \Gamma_{X} \longrightarrow \mathrm{Y}_{\Pi_{1}(X)}
$$

is an equivalence of 2-functors.

Proof. Fix a category C. By Propositions 1.2.5 and 1.2.6, it remains to show that $\mu_{X, C}$ is essentially surjective. Let us first suppose that $C$ is complete, hence we can work in the category of sheaves with values in C.

Consider a functor $\alpha: \Pi_{1}(X) \longrightarrow$ C. Define

$$
\mathcal{V}=\{(V, x) \mid x \in V, V \text { relatively 1-connected open subset of } X\}
$$

and set $(V, x) \leqslant(W, y)$ if and only if $W \subset V$, which turns $\mathcal{V}$ into a category.

Let $U \subset X$ be an open subset. We set

$$
\mathcal{F}_{\alpha}(U)=\lim _{\substack{(V, x) \in \mathcal{D} \\ V \subset U}} \alpha(x)
$$

where for any $(V, x) \leqslant(W, y)$ we chose a path $\gamma_{x y}: x \rightarrow y$ in $V$ and use the isomorphism $\alpha\left(\gamma_{x y}\right): \alpha(x) \rightarrow \alpha(y)$ in the projective system. Note that since $V$ is relatively 1-connected, this automorphism does not depend on the choice of $\gamma_{x y}$. Now let $U=\bigcup_{i \in I} U_{i}$ be a covering stable by finite intersection. In order to prove that $\mathcal{F}_{\alpha}$ is a sheaf, we have to show that the natural morphism

$$
\mathcal{F}_{\alpha}(U)=\lim _{\substack{(V, x) \in \mathcal{V} \\ V \subset U}} \alpha(x) \rightarrow \lim _{i \in I} \lim _{\substack{(V, x) \in \mathcal{V} \\ V \subset U_{i}}} \alpha(x)=\lim _{i \in I} \mathcal{F}_{\alpha}\left(U_{i}\right)
$$

is an isomorphism. To define the inverse isomorphism, note that for $(W, y) \in \mathcal{V}$ the isomorphisms $\alpha\left(\gamma_{x y}\right)^{-1}$ define the natural isomorphism

$$
\alpha(y) \stackrel{\sim}{\longrightarrow} \lim _{\substack{i,(V, x) \\ V \subset U_{i} \cap W}} \alpha(x) .
$$

Its inverse defines

$$
\lim _{\substack{i,(V, x) \\ V \subset U_{i}}} \alpha(x) \rightarrow \lim _{\substack{i,(V, x) \\ V \subset U_{i} \cap W}} \alpha(x) \simeq \alpha(y)
$$

which is compatible with the projective system.

By construction it is clear that, if $U$ is relatively 1-connected, then for any choice of $x \in U$ we get a natural isomorphism $\mathcal{F}_{\alpha}(U) \simeq \alpha(x)$ (this isomorphism being

${ }^{8}$ Recall that a topological space $X$ is locally relatively 1 -connected if each point $x \in X$ has a fundamental system of arcwise connected neighbourhoods $U$ such that each loop $\gamma$ in $U$ is homotopic in $X$ to a constant path. 
compatible with restrictions). Hence, since relatively 1-connected open subsets form a base of the topology of $X$, we get that $\mathcal{F}_{\alpha}$ is a locally constant sheaf that is constant on every relatively 1-connected open subset of $X$.

To calculate the monodromy, consider first a path $\gamma: x \rightarrow y$ such that there exists a relatively 1-connected open neighbourhood of $\gamma$. Obviously we get that $\mu\left(\mathcal{F}_{\alpha}\right)(\gamma)$ is naturally isomorphic to $\alpha(\gamma)$. For a general $\gamma$, we decompose $\gamma$ in a finite number of paths that can be covered by relatively 1-connected open subsets to get the result. Now the general case. Embed $\mathrm{C}$ into $\widehat{C}$ by the Yoneda functor

$$
Y: \mathrm{C} \longrightarrow \widehat{\mathrm{C}} \text {. }
$$

Then, given a representation $\alpha$, we can construct $\mathcal{F}_{\alpha}$ as a locally constant sheaf with values in $\widehat{\mathrm{C}}$. Then $\mathcal{F}_{\alpha}$ has representable stalks and is therefore in the essential image of the fully faithful functor

$$
\Gamma\left(X, C_{X}\right) \longrightarrow \Gamma\left(X, \widehat{C}_{X}\right) .
$$

Since by construction the monodromy of a locally constant sheaf with values in $C$ can be calculated by considering it as a locally constant sheaf with values in $\widehat{C}$, we can conclude.

Thanks to the 2-Yoneda lemma (as stated for example in [11, Chapter 1]), we immediately recover the following

Corollary 1.2.10. Let $X$ be locally relatively 1-connected. Then there is an equivalence of categories

$$
\Pi_{1}(X) \simeq \operatorname{Hom}\left(\Gamma_{X}, \operatorname{ld}_{\text {Cat }}\right)
$$

given by $x \mapsto \omega_{x}$.

\subsection{Degree 1 non abelian cohomology with constant coefficients}

Let $M$ be a (not necessarily commutative) monoid. Denote by $M[1]$ the small category with $\bullet$ as single object and $\operatorname{End}_{M[1]}(\bullet)=M$. Note that if $G$ is a group then $G[1]$ is a groupoid. Then it is easy to check that we get fully faithful functors of categories

$$
[1]: \text { Mon } \longrightarrow \text { Cat } \quad[1]: \mathrm{Gr} \longrightarrow \mathbf{G r},
$$

where we denote by Mon the category of small monoids and by Gr that of groups. Also note that if $G$ is a connected groupoid (that is, $\pi_{0}(G) \simeq \bullet$ ), then for each $P \in \mathrm{ObG}$, the inclusion functor $\operatorname{Aut}_{\mathrm{G}}(P)[1] \longrightarrow \mathrm{G}$ is an equivalence.

Consider the category $\operatorname{Set}(G)$ of right $G$-sets and $G$-linear maps. Then $G[1]$ is equivalent to the full sub-category of $\operatorname{Set}(G)$ with $G$ as single object. Hence the stack $G[1]_{X}$ is equivalent to the stack $\mathfrak{T o r s}\left(G_{X}\right)$ of (right) torsors over the constant sheaf $G_{X}{ }^{9}$.

${ }^{9}$ Recall that $\operatorname{Tors}\left(G_{X}\right)$ is the stack which associates to each open subset $U \subset X$ the category $\operatorname{Tors}\left(G_{U}\right)$ of right $G_{U}$-sheaves locally free of rank one. Note that $\operatorname{Tors}\left(G_{X}\right)$ is equivalent to the category of $G$-coverings over $X$. 
Assume that $X$ is locally relatively 1-connected. By Theorem 1.2.9 there is an equivalence of categories

$$
\operatorname{Tors}\left(G_{X}\right) \stackrel{\sim}{\longrightarrow} \operatorname{Hom}\left(\Pi_{1}(X), G[1]\right) .
$$

A standard cocycle argument shows that there is an isomorphism of pointed sets

$$
\pi_{0}\left(\operatorname{Tors}\left(G_{X}\right)\right) \stackrel{\sim}{\longrightarrow} H^{1}\left(X ; G_{X}\right) .
$$

Assume moreover that the space $X$ is connected, and let us calculate the pointed set $\pi_{0}\left(\operatorname{Hom}\left(\Pi_{1}(X), G[1]\right)\right)$. Since $\Pi_{1}(X)$ is connected, $\Pi_{1}(X)$ is equivalent to $\pi_{1}(X)[1]$ for a choice of a base point in $X$. Hence there is a natural surjective map

$$
\operatorname{Hom}_{\mathrm{Gr}}\left(\pi_{1}(X), G\right) \rightarrow \pi_{0}\left(\operatorname{Hom}\left(\pi_{1}(X)[1], G[1]\right)\right),
$$

and one checks immediately that two morphisms of groups $\varphi, \psi: \pi_{1}(X) \rightarrow G$ give isomorphic functors if and only if there exists $g \in G$ such that $\varphi=\operatorname{ad}(g) \circ \psi$, where $\operatorname{ad}(g)$ is the group automorphism of $G$ given by $h \mapsto g h g^{-1}$ for each $h \in G$ (automorphisms of this form are called inner automorphisms of $G$ ). Hence

$$
\operatorname{Hom}_{\mathrm{Gr}}\left(\pi_{1}(X), G\right) / G \simeq \pi_{0}\left(\operatorname{Hom}\left(\pi_{1}(X)[1], G[1]\right)\right)
$$

where $G$ acts on the left on $\operatorname{Hom}_{\mathrm{Gr}}\left(\pi_{1}(X), G\right)$ by conjugation. We get the classical

Proposition 1.3.1 (Hurewicz's formula). Let $X$ be connected and locally relatively 1-connected. Then for any group $G$ there is an isomorphism of pointed sets

$$
H^{1}\left(X ; G_{X}\right) \stackrel{\sim}{\longrightarrow} \operatorname{Hom}_{\mathrm{Gr}}\left(\pi_{1}(X), G\right) / G .
$$

In particular, if $G$ is commutative one recovers the isomorphism of groups

$$
H^{1}\left(X ; G_{X}\right) \stackrel{\sim}{\longrightarrow} \operatorname{Hom}_{\mathrm{Gr}}\left(\pi_{1}(X), G\right) .
$$

More generally, to each complex of groups $G^{-1} \stackrel{d}{\rightarrow} G^{0}$ one associates a small groupoid, which we denote by the same symbol, as follows: objects are the elements $g \in G^{0}$ and morphisms $g \rightarrow g^{\prime}$ are given by $h \in G^{-1}$ such that $d(h) g=g^{\prime}$. If moreover $G^{-1} \stackrel{d}{\rightarrow} G^{0}$ has the structure of crossed module ${ }^{10}$, the associated category is a strict $g r$-category, i.e. a group object in the category of groupoids ${ }^{11}$. In fact, all strict $g r$-categories arise in this way (see for example [5], and [SGA4, exposé XVIII] for the commutative case). In particular, if $G$ is a group, the groupoid $G[1]$ is identified with $G \rightarrow 1$ and it has the structure of a strict $g r$-category if and only if $G$ is commutative. Moreover, the strict $g r$-category Aut $(G[1])$ of auto-equivalences of $G[1]$ is equivalent to $G \stackrel{\text { ad }}{\longrightarrow} \operatorname{Aut}_{\mathrm{Gr}}(G)$.

Consider the constant stack $\left(G \stackrel{\text { ad }}{\longrightarrow} \operatorname{Aut}_{G r}(G)\right)_{X}$. It is equivalent to the $g r$-stack $\mathfrak{B i t o r s}\left(G_{X}\right)$ of $G_{X}$-bitorsors, i.e. $G_{X}$-torsors with an additional compatible structure of left $G_{X}$-torsors (see $[\mathbf{2}]$ for more details). Suppose that $X$ is locally relatively

${ }^{10}$ Recall that a complex of groups $G^{-1} \stackrel{d}{\rightarrow} G^{0}$ is a crossed module if it is endowed with a (left) action of $G^{0}$ on $G^{-1}$ such that (i) $d\left({ }^{g} h\right)=\operatorname{ad}(g)(d(h))$ and (ii) ${ }^{d(\tilde{h})} h=\operatorname{ad}(\tilde{h})(h)$ for any $h, \tilde{h} \in G^{-1}$ and $g \in G^{0}$. We use the convention as in [2] for which $G^{i}$ is in $i$-th degree.

${ }^{11}$ Precisely, the group-like structure is given by the following rule: $g \otimes g^{\prime}=g g^{\prime}$ for objects $g, g^{\prime} \in G^{0}$ and $h \otimes h^{\prime}=h^{g_{2}} h^{\prime}$ for arrows $g_{1} \stackrel{h}{\longrightarrow} g_{2}$ and $g_{1}^{\prime} \stackrel{h^{\prime}}{\longrightarrow} g_{2}^{\prime}$ in $G^{-1}$. 
1-connected. Then, by Theorem 1.2.9, there is an equivalence of $g r$-categories

$$
\operatorname{Bitors}\left(G_{X}\right) \stackrel{\sim}{\longrightarrow} \operatorname{Hom}\left(\Pi_{1}(X), G \stackrel{\text { ad }}{\longrightarrow} \operatorname{Aut}_{\mathrm{Gr}}(G)\right) .
$$

One may show (see loc. cit.) that

$$
\pi_{0}\left(\operatorname{Bitors}\left(G_{X}\right)\right) \simeq H^{0}\left(X ; G_{X} \stackrel{\text { ad }}{\longrightarrow} \operatorname{Aut}_{\mathrm{Gr}}\left(G_{X}\right)\right)
$$

where the right hand side is the 0-th (hyper-)cohomology group of $X$ with values in the sheaf of crossed modules $G_{X} \stackrel{\text { ad }}{\longrightarrow} \operatorname{Aut}_{G r}\left(G_{X}\right)$. Suppose that $X$ is connected. Then $\Pi_{1}(X) \simeq \pi_{1}(X)[1]$ and a similar calculation as above leads to the isomorphism of groups

$$
\pi_{0}\left(\operatorname{Hom}\left(\pi_{1}(X)[1], G \stackrel{\text { ad }}{\longrightarrow} \operatorname{Aut}_{\mathrm{Gr}}(G)\right)\right) \simeq \operatorname{Hom}_{\mathrm{Gr}}\left(\pi_{1}(X), \mathrm{Z}(G)\right) \rtimes \operatorname{Out}_{\mathrm{Gr}}(G),
$$

where $\mathrm{Z}(G)$ denotes the center of $G$ and $\operatorname{Out}_{\mathrm{Gr}}(G)=\operatorname{Aut}_{\mathrm{Gr}}(G) / G$ is the group of outer automorphisms of $G$, which acts on the left on $\operatorname{Hom}_{\mathrm{Gr}}\left(\pi_{1}(X), \mathrm{Z}(G)\right)$ by composition. We get

Proposition 1.3.2 (Hurewicz's formula II). Let $X$ be connected and locally relatively 1-connected. Then for any group $G$ there is an isomorphism of groups

$$
H^{0}\left(X ; G_{X} \stackrel{\text { ad }}{\longrightarrow} \operatorname{Aut}_{\mathrm{Gr}}\left(G_{X}\right)\right) \stackrel{\sim}{\longrightarrow} \operatorname{Hom}_{\mathrm{Gr}}\left(\pi_{1}(X), \mathrm{Z}(G)\right) \rtimes \operatorname{Out}_{\mathrm{Gr}}(G) .
$$

A similar result holds replacing $G \stackrel{\text { ad }}{\longrightarrow} \operatorname{Aut}_{\mathrm{Gr}}(G)$ by a general crossed module $G^{-1} \stackrel{d}{\rightarrow}$ $G^{0}$. More precisely, noticing that $\operatorname{ker} d$ is central in $G^{-1}$, one gets an isomorphism of groups

$$
H^{0}\left(X ; G_{X}^{-1} \stackrel{d}{\rightarrow} G_{X}^{0}\right) \stackrel{\sim}{\longrightarrow} \operatorname{Hom}_{\mathrm{Gr}}\left(\pi_{1}(X), \operatorname{ker} d\right) \rtimes \operatorname{coker} d .
$$

\section{Classification of locally constant stacks}

Following our presentation of 1-monodromy, we will approach the theory of 2monodromy in the setting of 2-stacks. We refer to [2] for the basic definitions in the theory of 2-stacks. Let $X$ be a topological space and let 2 Cat, $2 \mathbf{P S t}(X)$ and $2 \operatorname{St}(X)$ denote the 3 -category ${ }^{12}$ of small 2-categories, of 2-prestacks and that of 2-stacks on $X$, respectively. As for sheaves and stacks, there exists a 2-stack associated to a 2-prestack:

Proposition 2.0.3. The forgetful 3-functor

$$
\text { For : } 2 \operatorname{St}(X) \longrightarrow 2 \mathbf{P S t}(X)
$$

has a left adjoint 3-functor

$$
\ddagger: 2 \operatorname{PSt}(X) \longrightarrow 2 \operatorname{St}(X) .
$$

Hence, we may associate to any 2-category a constant 2-prestack on $X$ and set

${ }^{12}$ Here and in the following, we will use the terminology of 3-categories and 3-functors only in the framework of strict 3-categories, i.e. categories enriched in 2 Cat. 
Definition 2.0.4. Let $\mathbf{C}$ be a 2-category. The constant 2-stack on $X$ with stalk $\mathbf{C}$ is the image of $\mathbf{C}$ by the 3 -functor

$$
(\cdot)_{X}: 2 \text { Cat } \longrightarrow 2 \operatorname{PSt}(X) \stackrel{\ddagger}{\longrightarrow} 2 \operatorname{St}(X) .
$$

Objects in $\mathbf{C}_{X}(X)$ are called locally constant stacks on $X$ with values in $\mathbf{C}$, and 1-arrows in $\mathbf{C}_{X}(X)$ are called functors of locally constant stacks. A locally constant stack is constant with stalk $\mathrm{P}$, if it is equivalent to $\boldsymbol{\eta}_{X, \mathbf{C}}(\mathrm{P})$ for some object $\mathrm{P} \in$ $\mathrm{Ob} \mathbf{C}$, where the 2-functor

$$
\boldsymbol{\eta}_{X, \mathbf{C}}: \mathbf{C} \longrightarrow \mathbf{C}_{X}(X)
$$

is induced by the 3 -adjunction of Proposition 2.0.3.

Let us look at the case when $\mathbf{C}=\mathbf{C a t}$, the strict 2-category of all small categories. By the universal property of the 2-stack associated to a 2-prestack, the 2-functor which sends a category $C$ to the constant stack $C_{X}$ (see Definition 1.1.2) factors through $\boldsymbol{\eta}_{X, \text { Cat }}$. It follows that there is a fully faithful 2 -functor $\operatorname{Cat}_{X}(X) \longrightarrow$ $\operatorname{St}(X)$. It is easy to see that a stack $\mathfrak{S}$ on $X$ is in the essential image of this 2 -functor if and only if there exists an open covering $X=\bigcup U_{i}$ such that $\left.\mathfrak{S}\right|_{U_{i}}$ is equivalent to a constant stack. Hence $\operatorname{Cat}_{X}(X)$ is equivalent to the full 2-subcategory $\mathbf{L c S t}(X)$ of $\mathbf{S t}(X)$ whose objects are the stacks which are locally constant in the usual sense. More generally, let $\mathbf{C}$ be a 2-category which admits all the small 2-limits. Then one can define the notion of a stack with values in $\mathbf{C}$ analogous to the case of sheaves

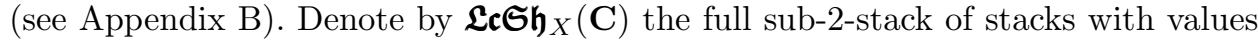
in $\mathbf{C}$ which are locally constant. It is then not difficult to prove the following

Proposition 2.0.5. Assume that $X$ is locally relatively 1-connected. Then there is a natural equivalence of 2-stacks

$$
\mathbf{C}_{X} \stackrel{\sim}{\longrightarrow} \mathfrak{L c S h}_{X}(\mathbf{C}) .
$$

For a more general $\mathbf{C}$, we can always embed $\mathbf{C}$ by the 2-Yoneda lemma into the strict 2-category $\widehat{\mathbf{C}}=\operatorname{Hom}\left(\mathbf{C}^{\mathrm{op}}, \mathbf{C a t}\right)$, which admits all small 2-limits (and 2-colimits). Then $\mathbf{C}_{X}(X)$ can be identified with the (essentially) full sub-2-category of $\widehat{\mathbf{C}}_{X}(X)$ defined by objects whose stalks are 2-representable.

We now follow Section 1 step by step to define the 2-monodromy 2 -functor.

Let $f: X \rightarrow Y$ be a continuous map. We leave to the reader to define the 3 -adjoint 3 -functors $f_{*}$ and $f^{-1}$.

Definition 2.0.6. Denote by $\boldsymbol{\Gamma}(X, \cdot)$ the 3 -functor of global sections

$$
2 \operatorname{St}(X) \longrightarrow 2 \text { Cat, } \quad \mathfrak{S} \mapsto \boldsymbol{\Gamma}(X, \mathfrak{S})=\mathfrak{S}(X)
$$

and set $\boldsymbol{\Gamma}_{X}=\boldsymbol{\Gamma}(X, \cdot) \circ(\cdot)_{X}$.

Since the 3 -functor

$$
\boldsymbol{\Gamma}(\{\mathrm{pt}\}, \cdot): 2 \mathbf{S t}(\{\mathrm{pt}\}) \longrightarrow 2 \mathbf{C a t}
$$

is an equivalence of 3 -categories, the 3 -functor $\boldsymbol{\Gamma}(X, \cdot)$ is right 3 -adjoint to $(\cdot)_{X}$. It is not hard to see that we get a 3 -transformation of 3 -functors $f^{-1}$ compatible 
with $\boldsymbol{\eta}_{X}$ and $\boldsymbol{\eta}_{Y}$, i.e. the following diagram commutes up to a natural invertible 2-modification:

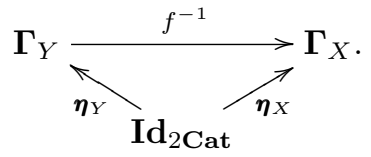

Similarly to the case of sheaves, this implies that for each point $x \in X$ and any locally constant stacks $\mathfrak{S}, \mathfrak{T} \in \mathbf{C}_{X}(X)$, the natural functor

$$
i_{x}^{-1} \mathfrak{H o m}_{\mathbf{C}_{X}}(\mathfrak{S}, \mathfrak{T}) \longrightarrow \operatorname{Hom}_{\mathbf{C}}\left(i_{x}^{-1} \mathfrak{S}, i_{x}^{-1} \mathfrak{T}\right)
$$

is an equivalence (here $i_{x}:\{\mathrm{pt}\} \rightarrow X$ denotes the natural map to $x$ and we identify $\mathbf{C}$ with global sections of $\mathbf{C}_{\mathrm{pt}}$ ). Therefore, for each continuous map $f: X \rightarrow Y$, we get a natural equivalence of locally constant stacks of categories

$$
f^{-1} \mathfrak{H o m}_{\mathbf{C}_{Y}}(\mathfrak{S}, \mathfrak{T}) \stackrel{\sim}{\longrightarrow} \mathfrak{H o m}_{\mathbf{C}_{X}}\left(f^{-1} \mathfrak{S}, f^{-1} \mathfrak{T}\right)
$$

\subsection{The 2-monodromy 2-functor}

Let us prove first the fundamental Lemma of homotopy invariance of locally constant stacks.

Lemma 2.1.1. Consider the maps $X \underset{p}{\stackrel{\iota_{t}}{\rightleftarrows}} X \times I$ as in Lemma 1.1.10. Then the 3-transformations

$$
\Gamma_{X} \underset{\iota_{t}^{-1}}{\stackrel{p^{-1}}{\longleftarrow}} \boldsymbol{\Gamma}_{X \times I}
$$

are equivalences of 3-functors, quasi-inverse one to each other.

Proof. Let $\mathbf{C}$ be a 2-category. Since $\iota_{t}^{-1} \circ p^{-1} \simeq \mathrm{Id}_{\boldsymbol{\Gamma}_{X}}$, it remains to check that for each locally constant stack $\mathfrak{S} \in \boldsymbol{\Gamma}\left(X \times I, \mathbf{C}_{X \times I}\right)$ there is a natural equivalence $p^{-1} \iota_{t}^{-1} \mathfrak{S} \simeq \mathfrak{S}$.

First, let us suppose that $\mathfrak{S}$ is a locally constant stack of categories and let us prove that the natural functor

$$
\iota_{t}^{-1}: \Gamma(X \times I, \mathfrak{S}) \longrightarrow \Gamma\left(X, \iota_{t}^{-1} \mathfrak{S}\right)
$$

is an equivalence. Since the sheaves $\mathcal{H}_{\mathfrak{S}}$ are locally constant, by Lemma 1.1.10 it is clear that this functor is fully faithful. Let us show that it is essentially surjective. Note that, since $\mathfrak{S}$ is locally constant, it is easy to see that for every open neighbourhood $U \times I_{j} \ni(x, t)$ such that $I_{j}$ is an interval and $\left.\mathfrak{S}\right|_{U \times I_{j}}$ is constant, there exists an open subset $\tilde{U} \ni x$ with the property that for every locally constant sheaf $\mathcal{F} \in \mathfrak{S}\left(\tilde{U} \times I_{j}\right)$ there exists $\tilde{\mathcal{F}} \in \mathfrak{S}(\tilde{U} \times I)$ such that $\left.\tilde{\mathcal{F}}\right|_{\tilde{U} \times I_{j}} \simeq \mathcal{F}$.

Now take $\mathcal{F} \in \Gamma\left(X, \iota_{t}^{-1} \mathfrak{S}\right)$. Then we can find a covering $X \times\{t\} \subset \bigcup_{j \in J} U_{j} \times I_{j}$ where $I_{j}$ are open intervals containing $t$ such that $\left.\mathfrak{S}\right|_{U_{j} \times I_{j}}$ is constant and we can find objects $\mathcal{F}_{j} \in \mathfrak{S}\left(U_{j} \times I_{j}\right)$ such that $\left.\iota_{t}^{-1} \mathcal{F}_{j} \simeq \mathcal{F}\right|_{U_{j}}$. Then the isomorphism

$$
\mathcal{H}_{\mathfrak{S}}\left(\left.\mathcal{F}_{i}\right|_{U_{i j} \times I_{i j}},\left.\mathcal{F}_{j}\right|_{U_{i j} \times I_{i j}}\right) \stackrel{\sim}{\longrightarrow} \mathcal{H} m_{\mathfrak{S}}\left(\left.\left.\mathcal{F}\right|_{U_{i}}\right|_{U_{i j}},\left.\left.\mathcal{F}\right|_{U_{j}}\right|_{U_{i j}}\right)
$$


implies that we may use the descent data of $\mathcal{F}$ to patch the $\mathcal{F}_{i}$ to a global object on $X \times I$ that is mapped to $\mathcal{F}$ by $\iota_{t}^{-1}$.

The rest of the proof is similar to that of Lemma 1.1.10. Let $\mathfrak{S} \in \mathbf{\Gamma}\left(X \times I, \mathbf{C}_{X \times I}\right)$ be a locally constant stack and consider the stack of functors $\mathfrak{H o m}_{\mathbf{C}_{X \times I}}\left(p^{-1} \iota_{t}^{-1} \mathfrak{S}, \mathfrak{S}\right)$. This is a locally constant stack of categories and the natural functor

$$
\iota_{t}^{-1} \mathfrak{H o m}_{\mathbf{C}_{X \times I}}\left(p^{-1} \iota_{t}^{-1} \mathfrak{S}, \mathfrak{S}\right) \longrightarrow \mathfrak{H o m}_{\mathbf{C}_{X}}\left(\iota_{t}^{-1} \mathfrak{S}, \iota_{t}^{-1} \mathfrak{S}\right)
$$

is an equivalence. We have thus shown that the natural functor

$$
\Gamma\left(X \times I, \mathfrak{H o m}_{\mathbf{C}_{X \times I}}\left(p^{-1} \iota_{t}^{-1} \mathfrak{S}, \mathfrak{S}\right)\right) \longrightarrow \Gamma\left(X, \mathfrak{H o m}_{\mathbf{C}_{X}}\left(\iota_{t}^{-1} \mathfrak{S}, \iota_{t}^{-1} \mathfrak{S}\right)\right)
$$

is an equivalence. We can therefore lift the identity of $\iota_{t}^{-1} \mathfrak{S}$ to get a functor

$$
p^{-1} \iota_{t}^{-1} \mathfrak{S} \stackrel{\sim}{\longrightarrow} \mathfrak{S}
$$

and it is not hard to prove that it is an equivalence.

Corollary 2.1.2. For each 2-category $\mathbf{C}$, the 2-functor

$$
\boldsymbol{\eta}_{I, \mathbf{C}}: \mathbf{C} \longrightarrow \boldsymbol{\Gamma}\left(I, \mathbf{C}_{I}\right)
$$

is an equivalence.

Hence, for any $X$ and any $t \in I$, we have the equivalence $\operatorname{ld}_{\boldsymbol{\Gamma}_{X}}=\left(p \circ \iota_{t}\right)^{-1} \simeq$ $\iota_{t}^{-1} p^{-1}$. Then, for any $s, t \in I$, there exists a canonical (i.e. unique up to unique invertible modification) equivalence $\iota_{t}^{-1} \simeq \iota_{s}^{-1}$. With patience, one deduces the following technical Lemma:

Lemma 2.1.3. (i) For any $s, t, t^{\prime} \in I$, the topological diagram on the left induces the diagram of equivalences on the right, which is commutative up to natural invertible modification:
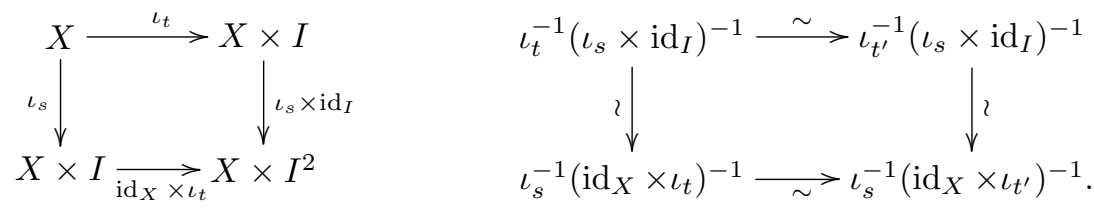

(ii) For any $r, s, t, t^{\prime} \in I$, the topological diagram

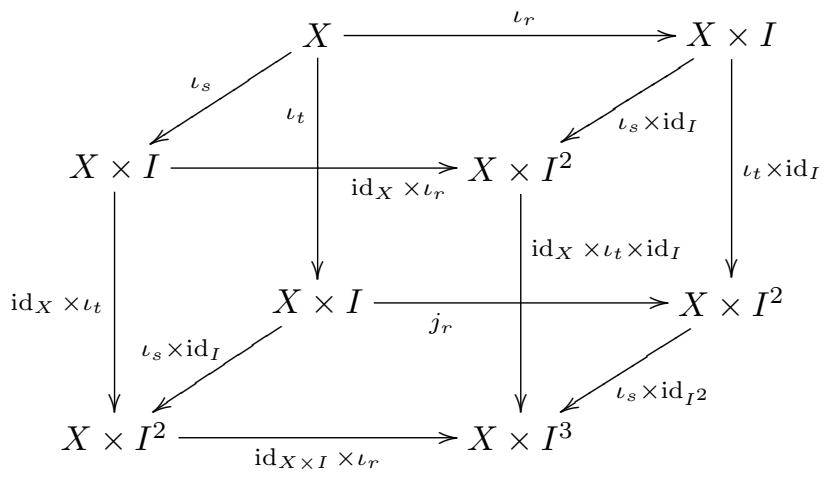


induces a (very big) commutative diagram of the corresponding modifications.

(iii) Let $f: X \rightarrow Y$ be a continuous map and $H_{f}: X \times I \rightarrow Y$ the constant homotopy of $f$. Then, for any $t, t^{\prime} \in I$, the diagram

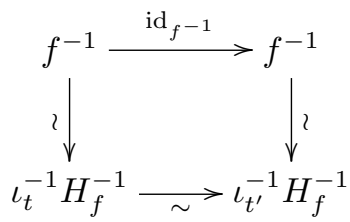

commutes up to natural invertible modification.

Let 2Top denote the 3-category whose objects are topological spaces, 1-arrows are continuous maps, 2-arrows are homotopies between continuous maps and 3arrows are homotopy classes of homotopies (between homotopies of maps). Following the same lines of the Proposition 1.1.13 and using the two Lemma above, one can then prove

Proposition 2.1.4. The assignment $(\mathbf{C}, X) \mapsto \boldsymbol{\Gamma}\left(X, \mathbf{C}_{X}\right)$ defines a 3-functor

$$
\Gamma: 2 \text { Cat } \times 2 \text { Top }^{\text {op }} \longrightarrow 2 \text { Cat },
$$

and the natural 2-functors $\boldsymbol{\eta}_{X, \mathrm{C}}$ define a 3-transformation $\boldsymbol{\eta}: \mathbf{Q}_{1} \longrightarrow \boldsymbol{\Gamma}$ (here $\mathbf{Q}_{1}$ denotes the canonical projection).

Definition 2.1.5. The homotopy 2-groupoid of $X$ is the 2 -groupoid ${ }^{13}$

$$
\Pi_{2}(X)=\operatorname{Hom}_{2 \mathbf{T o p}}(\{\mathrm{pt}\}, X) .
$$

Roughly speaking, its objects are the points of $X$ and for any $x, y \in X$, $\operatorname{Hom}_{\Pi_{2}(X)}(x, y)$ is the category $\Pi_{1}\left(P_{x, y} X\right)$, where $P_{x, y} X$ is the space of paths starting from $x$ and ending in $y$, endowed with the compact-open topology. Composition laws are defined in the obvious way. Note that, in particular, $\pi_{0}\left(\Pi_{2}(X)\right)=\pi_{0}(X)$, and for each $x \in X$ one has $\pi_{0}\left(\operatorname{Hom}_{\Pi_{2}(X)}(x, x)\right)=\pi_{1}(X, x)$ and $\operatorname{Hom}_{\mathrm{Hom}_{\Pi_{2}(X)}(x, x)}\left(\operatorname{id}_{x}, \operatorname{id}_{x}\right)=\pi_{2}(X, x)$. In general $\Pi_{2}(X)$ is not a strict 2category, and we refer to [10] for an explicit construction of a strictification when $X$ is Hausdorff.

Let $2 \mathrm{Gr}$ denote the 3 -category of 2 -groupoids. Then we have a 3 -functor

$$
\Pi_{2}: 2 \text { Top } \longrightarrow 2 \mathrm{Gr},
$$

which defines the Yoneda type 3-functor

$$
\mathbf{Y}_{\Pi_{2}}: 2 \text { Cat } \times 2 \text { Top }^{\text {op }} \longrightarrow 2 \text { Cat }, \quad(\mathbf{C}, X) \mapsto \operatorname{Hom}\left(\Pi_{2}(X), \mathbf{C}\right) .
$$

Definition 2.1.6. The 2-monodromy 3-transformation

$$
\boldsymbol{\mu}^{2}: \Gamma \longrightarrow \mathbf{Y}_{\Pi_{2}}
$$

${ }^{13}$ Recall that a 2 -groupoid is a 2 -category whose 2 -arrows are invertible and 1 -arrows are invertible up to a 2-arrow. 
is defined in the following manner. For each topological space $X$, the 2-functor

$$
\operatorname{Hom}_{2 \mathbf{T o p}}(\{\mathrm{pt}\}, X) \longrightarrow \operatorname{Hom}\left(\boldsymbol{\Gamma}\left(X, \mathbf{C}_{X}\right), \mathbf{C}\right)
$$

induces by evaluation a natural 2 -functor

$$
\boldsymbol{\Gamma}\left(X, \mathbf{C}_{X}\right) \times \Pi_{2}(X) \longrightarrow \mathbf{C},
$$

hence by adjunction a 2-functor

$$
\boldsymbol{\mu}_{X, \mathbf{C}}^{2}: \boldsymbol{\Gamma}\left(X, \mathbf{C}_{X}\right) \longrightarrow \operatorname{Hom}\left(\Pi_{2}(X), \mathbf{C}\right) .
$$

As in the case of 1-monodromy, let us visualize this construction using stalks. To every 2-stack $\mathfrak{S}$ we can associate its stalk at $x \in X$, which is the 2-category

$$
\mathfrak{S}_{x}=3 \lim _{x \in U} \mathfrak{S}(U)
$$

and a natural 2-functor $\boldsymbol{\rho}_{x, \mathfrak{S}}: \mathfrak{S}(X) \longrightarrow \mathfrak{S}_{x}$ (in the case that $\mathfrak{S}$ is the 2-stack $\mathfrak{S t}_{X}$ of all stacks on $X$, we can chose $\boldsymbol{\rho}_{x, \mathfrak{G t}}=\mathrm{F}_{x}$, the ordinary stalk 2-functor). Hence we get the natural stalk 3 -functor

$$
\mathbf{F}_{x}: 2 \mathbf{S t}(X) \longrightarrow 2 \mathbf{C a t} ; \quad \mathfrak{S} \mapsto \mathfrak{S}_{x}
$$

which induces an equivalence

$$
\mathfrak{L}_{\mathfrak{c S h}} \stackrel{\sim}{\longrightarrow} \text { Cat }
$$

(if $\mathbf{C}$ is a 2-category, then $\left(\mathbf{C}_{X}\right)_{x} \simeq \mathbf{C}$ ). Then one proves the following

Proposition 2.1.7. The assignment $(\mathbf{C},(X, x)) \mapsto\left(\mathbf{C}_{X}\right)_{x}$ defines a 3-functor

$$
\text { F : } 2 \text { Cat } \times 2 \text { Top }_{*}^{\text {op }} \longrightarrow 2 \text { Cat }
$$

and the 2-functors $\boldsymbol{\rho}_{x, \mathbf{C}}$ define a 3-transformation $\boldsymbol{\rho}: \boldsymbol{\Gamma} \longrightarrow \mathbf{F}$.

We find that

$$
\rho \circ \eta: \mathrm{Q}_{1} \longrightarrow \mathbf{F}
$$

is an equivalence of 3-functors. Let $\varepsilon: \mathbf{F} \longrightarrow \mathbf{Q}_{1}$ be a fixed quasi-inverse to $\boldsymbol{\rho} \circ \boldsymbol{\eta}$ and set $\boldsymbol{\omega}=\boldsymbol{\varepsilon} \circ \boldsymbol{\rho}$. Fix a topological space $X$ and a locally constant stack $\mathfrak{S} \in \boldsymbol{\Gamma}\left(X, \mathbf{C}_{X}\right)$. Then (up to a natural equivalence)

$$
\boldsymbol{\mu}_{X, \mathbf{C}}^{2}(\mathfrak{S})(x)=\omega_{x, \mathbf{C}}(\mathfrak{S})
$$

(if $\mathfrak{S}$ is a locally constant stack with values in Cat, then $\boldsymbol{\omega}_{x, \text { Cat }}(\mathfrak{S})$ can be canonically identified with $\mathfrak{S}_{x}$ ). If $\gamma: x \rightarrow y$ is a path, then the equivalence $\boldsymbol{\mu}_{X, \mathbf{C}}^{2}(\mathfrak{S})(\gamma)$ is defined by the chain of equivalences

$$
\boldsymbol{\omega}_{x, \mathbf{C}}(\mathfrak{S}) \simeq \boldsymbol{\omega}_{0, \mathbf{C}}\left(\gamma^{-1} \mathfrak{S}\right) \simeq \boldsymbol{\eta}_{I, \mathbf{C}}\left(\gamma^{-1} \mathfrak{S}\right) \simeq \boldsymbol{\omega}_{1, \mathbf{C}}\left(\gamma^{-1} \mathfrak{S}\right) \simeq \boldsymbol{\omega}_{y, \mathbf{C}}(\mathfrak{S}),
$$

where $\boldsymbol{\eta}_{I, \mathbf{C}}$ is just the global section functor in the case of ordinary stacks, i.e. for $\mathbf{C}=$ Cat. If $H: \gamma_{0} \rightarrow \gamma_{1}$ is a homotopy, then the invertible transformation 
$\boldsymbol{\mu}_{X, \mathbf{C}}^{2}(\mathfrak{S})(H)$ is defined by the diagram of equivalences

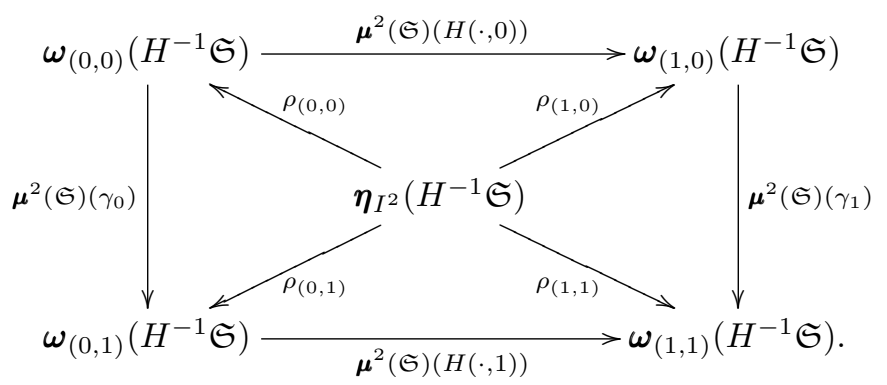

In particular, the following diagram of 3-transformations commutes (up to a natural 2-modification)

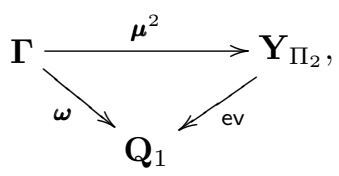

where ev denotes the evaluation 3-transformation.

\subsection{Classifying locally constant stacks}

Let $\Delta: \mathbf{Q}_{1} \longrightarrow \mathbf{Y}_{\Pi_{2}}$ denote the diagonal 3-transformation. Exactly as in the sheaf case, one gets

Proposition 2.2.1. The image of a constant stack is equivalent to a trivial representation. In other words, the diagram of 3-transformations

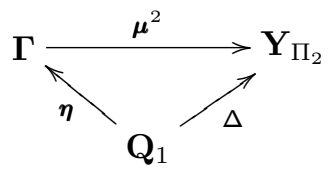

commutes up to invertible 2-modifications.

Proposition 2.2.2. For any topological space $X$ and 2-category $\mathbf{C}$, the 2-functor

$$
\boldsymbol{\mu}_{X, \mathbf{C}}^{2}: \boldsymbol{\Gamma}\left(X, \mathbf{C}_{X}\right) \longrightarrow \operatorname{Hom}\left(\Pi_{2}(X), \mathbf{C}\right)
$$

is faithful and conservative.

Proof. We have to show that, for any two locally constant stacks $\mathfrak{S}$ and $\mathfrak{S}^{\prime}$, the induced functor

$$
\boldsymbol{\mu}^{2}: \operatorname{Hom}_{\mathbf{C}_{X}}\left(\mathfrak{S}, \mathfrak{S}^{\prime}\right) \longrightarrow \operatorname{Hom}_{\mathbf{H o m}\left(\Pi_{2}(X), \mathbf{C}\right)}\left(\boldsymbol{\mu}^{2}(\mathfrak{S}), \boldsymbol{\mu}^{2}\left(\mathfrak{S}^{\prime}\right)\right)
$$

is faithful and conservative. Let $F, G: \mathfrak{S} \longrightarrow \mathfrak{S}^{\prime}$ be two functors. Since for each $x \in X$, there is a natural isomorphism $\operatorname{Hom}\left(\boldsymbol{\mu}^{2}(F)(x), \boldsymbol{\mu}^{2}(F)(x)\right) \simeq \operatorname{Hom}\left(F_{x}, G_{x}\right)$, 
we get the commutative diagram

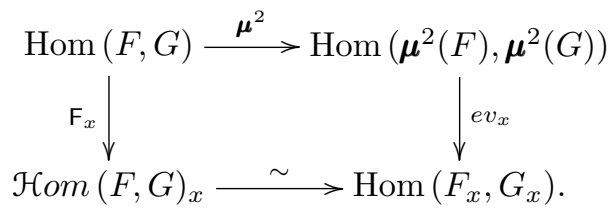

Let $\varphi, \psi: F \rightarrow G$ be two morphisms of functors. If $\boldsymbol{\mu}^{2}(\varphi)=\boldsymbol{\mu}^{2}(\psi)$, then $\varphi_{x}=\psi_{x}$ for all $x \in X$ and, since $\mathcal{H o m}(F, G)$ is a sheaf, we get $\varphi=\psi$. Similarly, if $\boldsymbol{\mu}^{2}(\varphi)$ is an isomorphism, it follows that the morphism $\varphi$ is an isomorphism.

Proposition 2.2.3. Let $X$ be locally 1-connected. Then for each 2-category $\mathbf{C}$, the 2-functor

$$
\boldsymbol{\mu}_{X, \mathbf{C}}^{2}: \boldsymbol{\Gamma}\left(X, \mathbf{C}_{X}\right) \longrightarrow \operatorname{Hom}\left(\Pi_{2}(X), \mathbf{C}\right)
$$

is full.

Proof. We have to show that the induced functor

$$
\boldsymbol{\mu}^{2}: \operatorname{Hom}_{\mathbf{C}_{X}}\left(\mathfrak{S}, \mathfrak{S}^{\prime}\right) \longrightarrow \operatorname{Hom}_{\mathbf{H o m}\left(\Pi_{2}(X), \mathbf{C}\right)}\left(\boldsymbol{\mu}^{2}(\mathfrak{S}), \boldsymbol{\mu}^{2}\left(\mathfrak{S}^{\prime}\right)\right)
$$

is full and essentially surjective.

A morphism $\phi: \boldsymbol{\mu}^{2}(\mathfrak{S}) \rightarrow \boldsymbol{\mu}^{2}\left(\mathfrak{S}^{\prime}\right)$ is defined by a family of functors

$$
\phi_{x}: \mathrm{ev}_{x} \boldsymbol{\mu}^{2}(\mathfrak{S}) \longrightarrow \mathrm{ev}_{x} \boldsymbol{\mu}^{2}\left(\mathfrak{S}^{\prime}\right)
$$

and for any path $\gamma: x \rightarrow x^{\prime}$ a canonical isomorphism

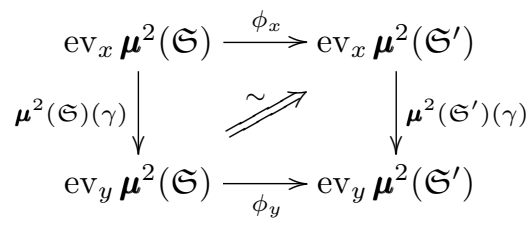

Since $\mathfrak{S}, \mathfrak{S}^{\prime}$ are locally constant, we have an equivalence of categories $\mathfrak{H o m}_{\mathbf{C}_{X}}\left(\mathfrak{S}, \mathfrak{S}^{\prime}\right)_{x} \simeq \operatorname{Hom}\left(\mathfrak{S}_{x}, \mathfrak{S}_{x}^{\prime}\right)$ and we may lift $\phi_{x}$ to a 1-connected open neighbourhood $U_{x}$ of $x$, say to a functor

$$
\varphi^{x}:\left.\left.\mathfrak{S}\right|_{U_{x}} \longrightarrow \mathfrak{S}^{\prime}\right|_{U_{x}}
$$

Consider $z \in U_{x} \cap U_{y}$ and chose paths $\gamma_{x z}$ from $x$ to $z$ and $\gamma_{y z}$ from $y$ to $z$. We get the diagram

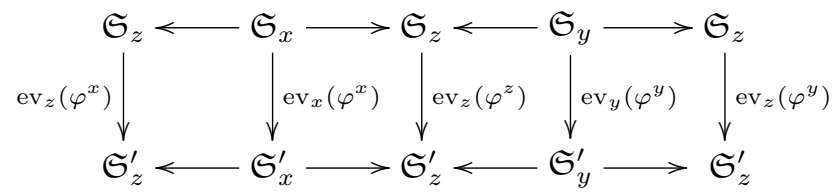

that commutes up to natural isomorphism. Moreover the horizontal lines are canonically isomorphic to identity morphisms, hence we get a natural isomorphism 
$\operatorname{ev}_{z}\left(\varphi^{x}\right) \simeq \operatorname{ev}_{z}\left(\varphi^{y}\right)$ that we can lift to a small neighbourhood of $z$. Since $U_{x}$ and $U_{y}$ are 1-connected, this lift does not depend on the choice of the paths $\gamma_{x z}$ and $\gamma_{y z}$ and the isomorphism $\operatorname{ev}_{z}\left(\varphi^{x}\right) \simeq \operatorname{ev}_{z}\left(\varphi^{y}\right)$ is canonical in a neighbourhood of $z$, i.e. it satisfies the cocycle condition and can be patched to an isomorphism

$$
\left.\left.\varphi^{x}\right|_{U_{x y}} \simeq \varphi^{y}\right|_{U_{x y}} .
$$

Clearly these isomorphisms satisfy the cocycle condition, hence we get a functor $\varphi: \mathfrak{S} \longrightarrow \mathfrak{S}^{\prime}$ By construction $\boldsymbol{\mu}^{2}(\varphi) \simeq \phi$

Next consider two functors $\varphi, \psi: \mathfrak{S} \longrightarrow \mathfrak{S}^{\prime}$ and a morphism $f: \boldsymbol{\mu}^{2}(\varphi) \rightarrow \boldsymbol{\mu}^{2}(\psi)$. Such a morphism is defined by a family

$$
f_{x}: \operatorname{ev}_{x} \boldsymbol{\mu}^{2}(\varphi) \rightarrow \mathrm{ev}_{x} \boldsymbol{\mu}^{2}(\psi)
$$

with compatibility conditions, hence by a family

$$
f_{x}: \varphi_{x} \rightarrow \psi_{x}
$$

such that for every path $\gamma$ from $x$ to $y$

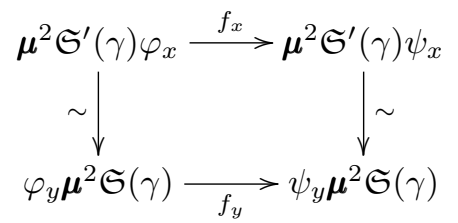

commutes. Lifting $f_{x}$ to any arcwise connected neighbourhood $U_{x}$ of $x$, this diagram implies that $\boldsymbol{\mu}^{2}\left(f_{x}\right)=f_{z}$ for all $z \in U_{z}$ and we can show that we can patch the $f_{x}$ in an analogous way as in the case of 1-monodromy.

Corollary 2.2.4. Let $X$ be 2-connected. Then the 2-functor

$$
\boldsymbol{\eta}_{X, \mathbf{C}}: \mathbf{C} \longrightarrow \mathbf{C}_{X}(X)
$$

is a natural equivalence of 2-categories. For $\mathbf{C}=\mathbf{C a t}$, the 2-functor $\Gamma(X, \cdot)$ provides a quasi-2-inverse to $\boldsymbol{\eta}_{X, \mathbf{C}}=(\cdot)_{X}$.

Proof. The proof follows the same lines as the proof of Proposition 1.2.7, using the fact that $\Pi_{2}(X)$ is trivial, that is, it reduces to the 2-category with a single object and only the identity 1 -arrow and 2-arrows. If $\mathbf{C}=\mathbf{C a t}$, one may chose $\boldsymbol{\eta}_{X, \mathbf{C}}=(\cdot)_{X}$, hence $\mathrm{F}_{x_{0}}$ gives a quasi-2-inverse for any choice of $x_{0} \in X$. Thanks to the natural 2-transformation $\Gamma(X, \cdot) \longrightarrow \mathrm{F}_{x_{0}}$, the 2-functor $\Gamma(X, \cdot)$ provides another quasi-2-inverse.

Theorem 2.2.5. Let $X$ be locally relatively 2-connected ${ }^{14}$. Then

$$
\mu_{X}^{2}: \Gamma_{X} \longrightarrow \mathbf{Y}_{\Pi_{2}(X)}
$$

is an equivalence of 3-functors.

\footnotetext{
${ }^{14}$ Recall that a topological space $X$ is locally relatively 2 -connected if each point $x \in X$ has a fundamental system of 1-connected neighbourhoods $U$ such that every homotopy of a path in $U$ is homotopic to the constant homotopy in $X$.
} 
Proof. We have to show that for each 2-category $\mathbf{C}$, the 2 -functor $\boldsymbol{\mu}_{X, \mathbf{C}}^{2}$ is essentially surjective.

Suppose first that $\mathbf{C}$ is 2-complete and let $\boldsymbol{\alpha} \in \operatorname{Hom}\left(\Pi_{2}(X)\right.$, Cat $)$. Set

$$
\mathcal{V}=\{(V, x) \mid x \in V, V \text { relatively 2-connected open subset of } X\}
$$

and define $(V, x) \leqslant(W, y)$ if and only if $W \subset V$.

Let $U \subset X$ be an open subset. We set

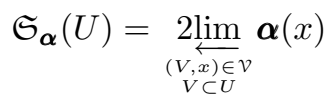

where for any $(V, x) \leqslant(W, y)$, we chose a path $\gamma_{x y}: x \rightarrow y$ in $V$ and use the equivalence $\boldsymbol{\alpha}\left(\gamma_{x y}\right): \boldsymbol{\alpha}(x) \stackrel{\sim}{\longrightarrow} \boldsymbol{\alpha}(y)$ in the projective system, and for any $(V, x) \leqslant$ $(W, y) \leqslant(Z, z)$, we chose a homotopy $H_{\gamma_{x y}, \gamma_{y z}, \gamma_{x z}}: \gamma_{x y} \gamma_{y z} \rightarrow \gamma_{x z}$ in $V$ and use the invertible transformation of functors $\boldsymbol{\alpha}\left(H_{\gamma_{x y}, \gamma_{y z}, \gamma_{x z}}\right)$. Note that since $V$ is relatively 2-connected, the equivalence $\boldsymbol{\alpha}\left(\gamma_{x y}\right)$ is unique up to invertible transformation and the invertible transformation $\boldsymbol{\alpha}\left(H_{\gamma_{x y}, \gamma_{y z}, \gamma_{x z}}\right)$ does not depend on the choice of the homotopy $H_{\gamma_{x y}, \gamma_{y z}, \gamma_{x z}}$.

One argues as in the proof of Theorem 1.2.9 to show that the pre-stack defined by $X \supset U \mapsto \mathfrak{S}_{\boldsymbol{\alpha}}(U)$ is actually a stack. By definition it is clear that, if $U$ is relatively 2-connected, then for any choice of $x \in U$ (and paths from $x$ to $y$ for every $y \in U$ ) we get an equivalence of categories $\mathfrak{S}_{\boldsymbol{\alpha}}(U) \simeq \boldsymbol{\alpha}(x)$ compatible with restriction functors in a natural sense. Hence, the stack $\mathfrak{S}_{\boldsymbol{\alpha}}$ is constant on every relatively 2 -connected open subset of $X$. Since relatively 2-connected open subsets form a base of the topology of $X$, we get that $\mathfrak{S}_{\boldsymbol{\alpha}}$ is locally constant.

The computation of the 2-monodromy of $\mathfrak{S}_{\boldsymbol{\alpha}}$ is similar to that of 1-monodromy in the proof of Theorem 1.2.9.

For a general 2-category $\mathbf{C}$, we can use the 2-Yoneda lemma to reduce to this last case.

Suppose that $X$ is connected and locally relatively 2-connected, and let $\Omega X$ denote the loop space $P_{x_{0}, x_{0}} X$ of paths starting and ending at a fixed base point $x_{0} \in X$. Consider the following diagram of topological space and continuous maps

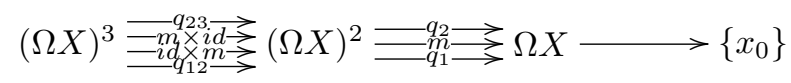

where the $q_{i}$ 's, the $q_{i j}$ 's and the $q_{i j k}$ 's are the natural projections and $m$ the composition of paths in $\Omega X^{15}$.

Let $\mathfrak{S}$ be a locally constant stack on $X$ with values in $\mathbf{C}$. Theorem 2.2 .5 asserts that $\mathfrak{S}$ is completely and uniquely (up to equivalence) determined by its 2 monodromy $\boldsymbol{\mu}_{X, \mathbf{C}}^{2}(\mathfrak{S}): \Pi_{2}(X) \longrightarrow \mathbf{C}$. Since $X$ is connected, the stalks of $\mathfrak{S}$ are all equivalent. Let us denote by $\mathrm{P}$ the stalk at $x_{0}$. By choosing paths from $x_{0}$ to

${ }^{15}$ Note that $\Omega X$ does not define a simplicial topological space, since the maps $m \circ(i d \times m)$ and $m \circ(m \times i d)$ are not equal but only homotopic. What one gets is a 2 -simplicial object in the 2 -category Top. This will not cause particular difficulties, since for locally constant objects there is a natural invertible transformation of functors $(m \times i d)^{-1} m^{-1} \stackrel{\sim}{\longrightarrow}(i d \times m)^{-1} m^{-1}$. 
any point $x$, the 2 -monodromy reads as a monoidal functor $\boldsymbol{\mu}_{X, \mathbf{C}}^{2}(\mathfrak{S}): \Pi_{1}(\Omega X) \longrightarrow$ Aut $_{\mathbf{C}}(\mathrm{P})$, where Aut $_{\mathbf{C}}(\mathrm{P})$ is the monoidal category of auto-equivalences of $\mathrm{P}$ in $\mathbf{C}$. Since the topological space $\Omega X$ satisfies the hypothesis of Theorem 1.2 .9 , there is a chain of equivalences of categories

$$
\operatorname{Hom}\left(\Pi_{1}(\Omega X), \operatorname{Aut}_{\mathbf{C}}(\mathrm{P})\right) \underset{\mu}{\stackrel{\sim}{\sim}} \Gamma\left(\Omega X, \operatorname{Aut}_{\mathbf{C}}(\mathrm{P})_{\Omega X}\right) \simeq \operatorname{Aut}_{\mathbf{C}_{X}}\left(\boldsymbol{\eta}_{\Omega X, \mathbf{C}}(\mathrm{P})\right) \text {. }
$$

Then the 2-monodromy is equivalent to a pair $(\alpha, \nu)$ where $\alpha: \boldsymbol{\eta}_{\Omega X, \mathbf{C}}(\mathrm{P}) \stackrel{\sim}{\longrightarrow}$ $\boldsymbol{\eta}_{\Omega X, \mathbf{C}}(\mathrm{P})$ is an equivalence of constant stacks on $\Omega X$ and

$$
\nu: q_{1}^{-1} \alpha \circ q_{2}^{-1} \alpha \stackrel{\sim}{\rightarrow} m^{-1} \alpha
$$

is an invertible transformation of functors of stacks on $(\Omega X)^{2}$ such that the following diagram of invertible transformations of functors of stacks on $(\Omega X)^{3}$ commutes

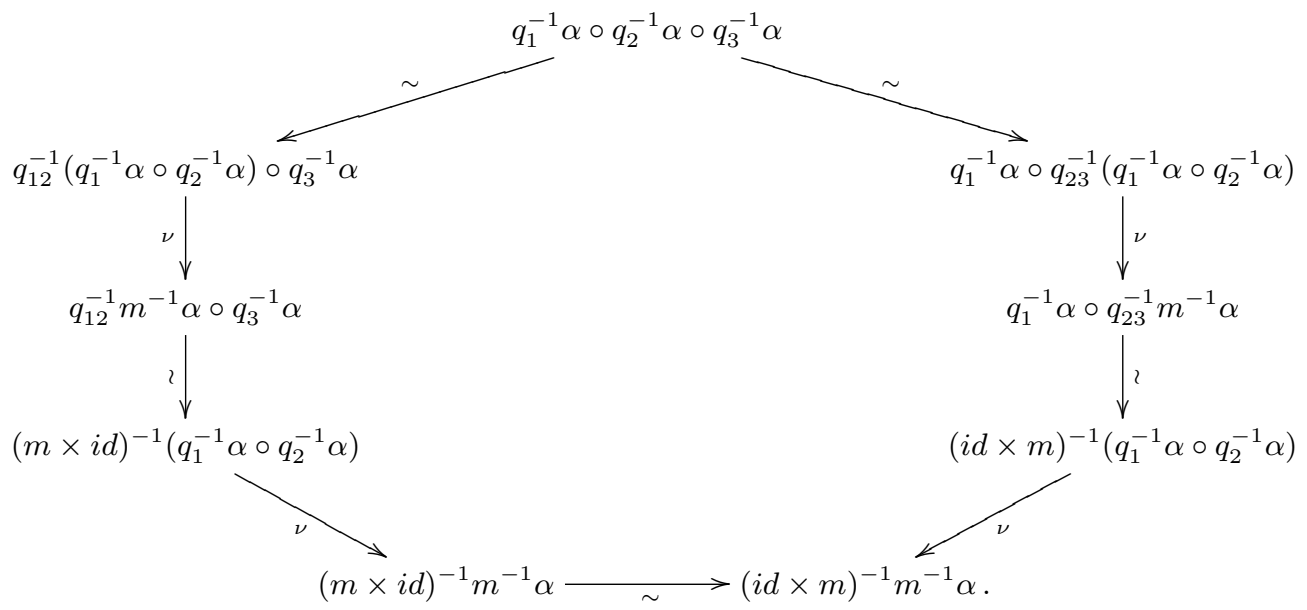

Roughly speaking, $\nu$ is given by a family of functorial invertible transformations $\nu_{12}: \alpha_{\gamma_{1}} \circ \alpha_{\gamma_{2}} \stackrel{\sim}{\longrightarrow} \alpha_{\gamma_{1} \gamma_{2}}$ for any $\gamma_{1}, \gamma_{2} \in \Omega X$, such that for $\gamma_{1}, \gamma_{2}, \gamma_{3} \in \Omega X$ the following diagram commutes

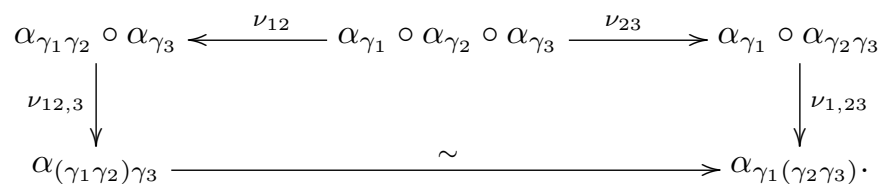

Definition 2.2.6. We call the triplet $(\mathrm{P}, \alpha, \nu)$ a descent datum for the locally constant stack $\mathfrak{S}$ on $X$.

Let us analyse a particular case, for which the descent datum admits a more familiar description. Let $G$ be a (not necessarily commutative) group. Recall that a $G_{X}$-gerbe is a stack locally equivalent to the stack of torsors $\mathfrak{T o r s}\left(G_{X}\right) \simeq G[1]_{X}$, that is, a locally constant stack on $X$ with stalk the groupoid $G[1]$. By the Morita theorem for torsors (see [9, Chapitre IV]), an equivalence $\alpha: G[1]_{\Omega X} \stackrel{\sim}{\longrightarrow} G[1]_{\Omega X}$ is given by $\mathcal{N} \mapsto \mathcal{P} \wedge \mathcal{N}$ for a $G_{\Omega X}$-bitorsor $\mathcal{P}$, where $\cdot \wedge \cdot$ denotes the contracted 
product.

Let $\mathfrak{G}$ be a $G_{X}$-gerbe and assume that $X$ is connected and locally relatively 2connected. Hence a descent datum for $\mathfrak{G}$ is given by the datum of $(G[1], \mathcal{P}, \nu)$, where $\mathcal{P}$ is a $G_{\Omega X}$-bitorsor and

$$
\nu: q_{1}^{-1} \mathcal{P} \wedge q_{2}^{-1} \mathcal{P} \stackrel{\sim}{\rightarrow} m^{-1} \mathcal{P}
$$

is an isomorphism of $G_{\Omega X}$-bitorsors on $(\Omega X)^{2}$ satisfying a commutative constraint similar to that of diagram (2.2.1). We refer to [6, Chapter 6$]$, for related constructions of line bundles on the free loop space of a manifold.

\subsection{Degree 2 non abelian cohomology with constant coefficients}

Let us fix some notations first. Recall that for each object $\mathrm{Q}$ of a 2-category $\mathbf{C}$, we denote by Aut $_{\mathbf{C}}(\mathrm{Q})$ the monoidal category of auto-equivalences of $\mathrm{Q}$ in $\mathbf{C}$. We set ${ }^{16}$ $\operatorname{Pic}_{\mathbf{C}}(\mathrm{Q})=\pi_{0}\left(\operatorname{Aut}_{\mathbf{C}}(\mathrm{Q})\right)$ and $\mathrm{Z}_{\mathbf{C}}(\mathrm{Q})=\operatorname{Aut}_{\text {Aut }_{\mathbf{C}}(\mathrm{Q})}\left(\operatorname{id}_{\mathrm{Q}}\right)$. Note that they both have a group structure (the latter being commutative) and that $\operatorname{Pic}_{\mathbf{C}}(\mathrm{Q})$ acts on $\mathrm{Z}_{\mathbf{C}}(\mathrm{Q})$ by conjugation. Explicitly, if $F \in$ Aut $_{\mathbf{C}}(\mathrm{Q})$, the action is induced by composition of the group isomorphisms (given by left and right vertical composition with $\operatorname{id}_{F}$ )

$$
\mathrm{Z}_{\mathbf{C}}(\mathrm{Q}) \stackrel{\sim}{\rightarrow} \operatorname{Aut}_{\text {Aut }_{\mathbf{C}}(\mathrm{Q})}(F) \stackrel{\sim}{\leftarrow} \mathrm{Z}_{\mathbf{C}}(\mathrm{Q}),
$$

since one easily checks that this depends only on the isomorphism class of $F$. If there is no risk of confusion, for a category (resp. $G$-linear category) C, we will use the shorter notations Aut (C), Pic(C) (resp. Aut $\left.{ }_{G}(\mathrm{C}), \operatorname{Pic}_{G}(\mathrm{C})\right)$ and $\mathrm{Z}(\mathrm{C})$. The latter group is usually called the center of $C$. As an example, for each $x \in X$ one has $\operatorname{Pic}_{\Pi_{2}(X)}(x)=\pi_{1}(X, x)$ and $Z_{\Pi_{2}(X)}(x)=\pi_{2}(X, x)$, and the action of $\pi_{1}(X, x)$ on $\pi_{2}(X, x)$ above described is exactly the classical one in algebraic topology.

Let $\mathrm{M}$ be a monoidal category. Denote by $\mathrm{M}[1]$ the 2-category with $\bullet$ as single object and $\operatorname{End}_{\mathrm{M}[1]}(\bullet)=\mathrm{M}$. Note that, if $\mathrm{M}$ is a groupoid whose monoidal structure is rigid ${ }^{17}$, then $M[1]$ is a 2 -groupoid. It is easy to see that we get a fully faithful 2 -functor

$$
\text { [1]: Mon } \longrightarrow 2 \text { Cat, }
$$

where we denote by Mon the strict 2-category of small monoidal categories with monoidal functors and monoidal transformations. This functor sends rigid monoidal groupoids to 2-groupoids. We follow $[\mathbf{3}]$ and call $g r$-category a rigid monoidal groupoid.

Note that if $M$ is a monoid, then $M[1]$ is monoidal if and only if $M$ is commutative, and if $M$ is also a group, then $M[1]$ is a $g r$-category. Hence we get fully faithful functors of categories

$$
[2]=[1] \circ[1]: \text { Mon }^{c} \longrightarrow \text { Mon } \longrightarrow 2 \text { Cat }, \quad[2]: \mathrm{Gr}^{\mathrm{c}} \longrightarrow 2 \mathbf{G r}
$$

\footnotetext{
${ }^{16}$ This is consistent with the classical notion of Picard group. Indeed, if $R$ is a ring and $\operatorname{Mod}(R)$ the $\left(\mathbb{Z}\right.$-linear) category of its left modules, by the Morita theorem the $\operatorname{group} \operatorname{Pic}_{\mathbf{C a t}_{\mathbb{Z}}}(\operatorname{Mod}(R))$ is isomorphic to the Picard group of $R$.

${ }^{17}$ Recall that a monoidal category $(\mathrm{M}, \otimes, I)$ is rigid if for each $P \in \mathrm{Ob}$ M there exists an object $Q$ and natural isomorphisms $P \otimes Q \simeq I$ and $Q \otimes P \simeq I$.
} 
where the uppercase c means commutative structures. Conversely, if $\mathbf{G}$ is a connected 2-groupoid, for each object $\mathrm{P} \in \mathrm{Ob} \mathbf{G}$, the inclusion 2-functor $\operatorname{Aut}_{\mathbf{G}}(\mathrm{P})[1] \longrightarrow$ $\mathbf{G}$ is a 2-equivalence. If $\mathbf{G}$ is even 1-connected (i.e. moreover $\operatorname{Aut}_{\mathbf{G}}(\mathrm{P})$ is a connected groupoid for some, hence all, $\mathrm{P})$, then $\mathrm{Z}_{\mathbf{G}}(\mathrm{P})[2] \simeq \mathbf{G}$.

For a not necessarily commutative group $G$, we can consider the strict $g r$-category Aut $(G[1])$ which gives rise to the 2-groupoid

$$
G \llbracket 2 \rrbracket=\operatorname{Aut}(G[1])[1] .
$$

Recall that Aut $(G[1])$ is equivalent to $G \stackrel{\text { ad }}{\longrightarrow} \operatorname{Aut}_{\mathrm{Gr}}(G)$. Hence if $G$ is commutative, then Aut $(G[1])$ is completely disconnected but only id $\in$ Ob Aut $(G[1])$ is $G$-linear, so we get a monoidal functor

$$
\text { Aut }_{G}(G[1])[1] \simeq G[2] \longrightarrow G \llbracket 2 \rrbracket=\text { Aut }(G[1])[1]
$$

that identifies $G[2]$ to a sub-2-category of $G \llbracket 2 \rrbracket$ which has only the identity 1-arrow but the same 2-arrows.

Consider an object $C$ of the 2-category Cat (resp. Cat ${ }_{G}$ ). Then End (C)[1] (resp. End $_{G}(\mathrm{C})[1]$ ) is just the full sub-2-category of Cat (resp. Cat ${ }_{G}$ ) with the single object C. Hence, End $(C)[1]_{X}\left(\right.$ resp. End $\left.{ }_{G}(C)[1]_{X}\right)$ is the 2-stack of locally constant stacks (resp. $G_{X}$-linear stacks) on $X$ with stalk C.

If $X$ is a locally relatively 2 -connected space, by Theorem 2.2 .5 equivalence classes of such stacks are classified by the set

$$
\pi_{0}\left(\operatorname{Hom}\left(\Pi_{2}(X), \text { Aut }(\mathrm{C})[1]\right)\right) \text {. }
$$

Assume moreover that $X$ is connected. Then the 2-groupoid $\Pi_{2}(X)$ is connected, hence it is equivalent to $\Pi_{1}(\Omega X)[1]$ for some base point $x_{0} \in X$. Hence there is a natural surjective map

$$
\pi_{0}\left(\operatorname{Hom}_{\otimes}\left(\Pi_{1}(\Omega X), \operatorname{Aut}(\mathrm{C})\right)\right) \longrightarrow \pi_{0}\left(\operatorname{Hom}\left(\Pi_{1}(\Omega X)[1], \text { Aut }(\mathrm{C})[1]\right)\right),
$$

where $\operatorname{Hom}_{\otimes}(\cdot, \cdot)$ denotes the category of monoidal functors. One checks that, given two monoidal functors $\Phi, \Psi: \Pi_{1}(\Omega X) \longrightarrow$ Aut $(\mathrm{C})$, they give equivalent 2-functors if and only if there exists an equivalence $\varphi: C \stackrel{\sim}{\longrightarrow} \mathrm{C}$ and an invertible monoidal transformation $\alpha: \Phi(\cdot) \circ \varphi \stackrel{\sim}{\longrightarrow} \varphi \circ \Psi(\cdot)$. We thus get that the set (2.3.1) is isomorphic to

$$
\pi_{0}\left(\operatorname{Hom}_{\otimes}\left(\Pi_{1}(\Omega X), \operatorname{Aut}(\mathrm{C})\right)\right) / \operatorname{Pic}(\mathrm{C}),
$$

where the group $\operatorname{Pic}(C)$ acts by conjugation. A similar result holds, replacing Cat by $\mathbf{C a t}_{G}$.

The previous classification becomes very simple in the following case:

Proposition 2.3.1. Let $X$ be connected and locally relatively 2-connected. If C is a category with trivial center, then the set of equivalence classes of locally constant stacks on $X$ with stalk $\mathrm{C}$ is isomorphic to $H^{1}\left(X ; \operatorname{Pic}(\mathrm{C})_{X}\right)$.

Proof. Suppose for simplicity that $\mathrm{C}$ is a groupoid. Since $\mathrm{Z}(\mathrm{C}) \simeq 1$, the monoidal functor Aut $(\mathrm{C}) \stackrel{\pi_{0}}{\longrightarrow} \mathrm{Pic}(\mathrm{C})[0]$ is an equivalence, where the group is considered as a discrete category. Hence it easy to check that

$$
\pi_{0}\left(\operatorname{Hom}_{\otimes}\left(\Pi_{1}(\Omega X), \operatorname{Pic}(\mathrm{C})[0]\right)\right) \simeq \operatorname{Hom}_{\mathrm{Gr}}\left(\pi_{1}(X), \operatorname{Pic}(\mathrm{C})\right) .
$$


Hence $\boldsymbol{\pi}_{0}\left(\operatorname{Hom}\left(\Pi_{2}(X)\right.\right.$, Aut $\left.\left.(C)[1]\right)\right)$ is isomorphic to $\operatorname{Hom}_{\mathrm{Gr}}\left(\pi_{1}(X), \operatorname{Pic}(\mathrm{C})\right) / \mathrm{Pic}(\mathrm{C})$, where $\operatorname{Pic}(\mathrm{C})$ acts by conjugation. It remains to apply Proposition 1.3.1.

Let us analyse more in detail the case of gerbes. (References are made to $[\mathbf{9 , 2}, \mathbf{6}]$.) We start with the abelian case. Let $G$ be a commutative group and take $\mathrm{C}=G[1]$. Since there is an obvious equivalence of strict $g r$-categories $G[1] \simeq \operatorname{End}_{G}(G[1])$, it follows that $G[2]$ is just the full sub-2-category of $\mathbf{C a t}_{G}$ with the single object $G[1]$. Hence the 2-category $\boldsymbol{\Gamma}\left(X, G[2]_{X}\right)$ is equivalent to the strict 2-category $\mathbf{G e r}_{a b}\left(G_{X}\right)$ of abelian $G_{X^{-}}$-gerbes, that is, $G_{X^{-}}$-linear stacks locally $G_{X^{-}}$-equivalent to the $G_{X^{-}}$ linear stack of torsors $\mathfrak{T o r s}\left(G_{X}\right) \simeq G[1]_{X}$.

By some cocycle arguments (see for example $[6$, Chapter IV]), one shows that there is an isomorphism of groups

$$
\pi_{0}\left(\operatorname{Ger}_{a b}\left(G_{X}\right)\right) \simeq H^{2}\left(X ; G_{X}\right) .
$$

Assume that $X$ is locally relatively 2 -connected. By Theorem 2.2.5, there is an equivalence of monoidal 2-categories

$$
\operatorname{Ger}_{a b}\left(G_{X}\right) \stackrel{\sim}{\longrightarrow} \operatorname{Hom}\left(\Pi_{2}(X), G[2]\right) .
$$

Since $\operatorname{Pic}_{G}(G[1]) \simeq 1$, if $X$ is connected $(2.3 .2)$ gives the group $\pi_{0}\left(\operatorname{Hom}_{\otimes}\left(\Pi_{1}(\Omega X)\right.\right.$, $G[1]))$. Hence we get

Proposition 2.3.2 (Hurewicz-Hopf's formula). Let $X$ be connected and locally relatively 2-connected. Then for any commutative group $G$ there is an isomorphism of groups

$$
H^{2}\left(X ; G_{X}\right) \stackrel{\sim}{\longrightarrow} \pi_{0}\left(\operatorname{Hom}_{\otimes}\left(\Pi_{1}(\Omega X), G[1]\right)\right) .
$$

To give an explicit description of the right hand side, let us start by considering a $g r$-category H. Recall that there exists an "essentially exact" 18 sequence of $g r$ categories

$$
1 \longrightarrow \mathrm{A}_{\mathrm{H}}[1] \stackrel{i}{\longrightarrow} \mathrm{H} \stackrel{\pi_{0}}{\longrightarrow} \pi_{0}(\mathrm{H})[0] \longrightarrow 1,
$$

where $A_{H}$ denotes the commutative group $\operatorname{Aut}_{H}(I)$ of automorphims of the unit object, and the group $\pi_{0}(\mathrm{H})$ is considered as a discrete category. Then, if $G$ is another $g r$-category, a direct computation shows that there is an exact sequence of pointed sets

$$
1 \rightarrow \pi_{0}\left(\mathrm{Hom}_{\otimes}\left(\pi_{0}(\mathrm{H})[0], \mathrm{G}\right)\right) \rightarrow \pi_{0}\left(\mathrm{Hom}_{\otimes}(\mathrm{H}, \mathrm{G})\right) \rightarrow \pi_{0}\left(\mathrm{Hom}_{\otimes}\left(\mathrm{A}_{\mathrm{H}}[1], \mathrm{G}\right)\right) .
$$

Lemma 2.3.3. Let $\mathrm{G}=G[1]$, for an abelian group $G$. Then (2.3.3) gives an exact sequence of abelian groups

$$
1 \rightarrow H^{2}\left(\pi_{0}(\mathrm{H}) ; G\right) \rightarrow \pi_{0}\left(\mathrm{Hom}_{\otimes}(\mathrm{H}, G[1])\right) \rightarrow \operatorname{Hom}_{\mathrm{Gr}}\left(\mathrm{A}_{\mathrm{H}}, G\right),
$$

where $G$ is view as a $\pi_{0}(\mathrm{H})$-module with trivial action.

${ }^{18}$ This means that the monoidal functor $i$ (resp. $\pi_{0}$ ) is fully faithful (resp. essentially surjective) and that the essential image of $i$ is equivalent to the kernel of $\pi_{0}$ as monoidal categories. 
Proof. Set $H=\pi_{0}(\mathrm{H})$. It is easy to see that a monoidal functor $H[0] \longrightarrow G[1]$ is given by a set-theoretic function $\lambda: H \times H \rightarrow G$ such that

$$
\lambda\left(h_{1}, h_{2}\right) \lambda\left(h_{1} h_{2}, h_{3}\right)=\lambda\left(h_{2}, h_{3}\right) \lambda\left(h_{1}, h_{2} h_{3}\right),
$$

and that two monoidal functors $\lambda, \lambda^{\prime}$ are isomorphic if and only if there exists a function $\nu: H \rightarrow G$ such that

$$
\lambda\left(h_{1}, h_{2}\right) \nu\left(h_{1} h_{2}\right)=\lambda^{\prime}\left(h_{1}, h_{2}\right) \nu\left(h_{1}\right) \nu\left(h_{2}\right) .
$$

Hence we get an isomorphism of groups

$$
\pi_{0}\left(\mathrm{Hom}_{\otimes}(H[0], G[1]) \simeq H^{2}(H ; G),\right.
$$

where $G$ is view as a $H$-module with trivial action.

Similarly, one easily checks that $\pi_{0}\left(\mathrm{Hom}_{\otimes}\left(\mathrm{A}_{\mathrm{H}}[1], G[1]\right)\right)$ is isomorphic to $\operatorname{Hom}_{\mathrm{Gr}}\left(\mathrm{A}_{\mathrm{H}}, G\right)$.

Let $\operatorname{Hom}_{\pi_{0}(\mathrm{H})}\left(\mathrm{A}_{\mathrm{H}}, G\right)$ denote the subgroup of morphisms in $\operatorname{Hom}_{\mathrm{Gr}}\left(\mathrm{A}_{\mathrm{H}}, G\right)$ which are $\pi_{0}(\mathrm{H})$-linear with respect to the conjugation action ${ }^{19}$ on $\mathrm{A}_{\mathrm{H}}$ and to the trivial one on $G$. One easily checks that the morphism $\pi_{0}\left(\operatorname{Hom}_{\otimes}(\mathrm{H}, G[1])\right) \rightarrow \operatorname{Hom}_{G r}\left(\mathrm{~A}_{\mathrm{H}}, G\right)$ factors through $\operatorname{Hom}_{\pi_{0}(\mathrm{H})}\left(\mathrm{A}_{\mathrm{H}}, G\right)$. Then one gets an exact sequence of abelian groups

$$
1 \rightarrow H^{2}\left(\pi_{0}(\mathrm{H}) ; G\right) \rightarrow \pi_{0}\left(\operatorname{Hom}_{\otimes}(\mathrm{H}, G[1])\right) \rightarrow \operatorname{Hom}_{\pi_{0}(\mathrm{H})}\left(\mathrm{A}_{\mathrm{H}}, G\right) \stackrel{\delta}{\rightarrow} H^{3}\left(\pi_{0}(\mathrm{H}) ; G\right)
$$

where the coboundary morphism $\delta$ is described as follows.

Recall that to the $g r$-category $\mathrm{H}$ one associates a cohomology class $^{20}[\mathrm{H}]$ in $H^{3}\left(\pi_{0}(\mathrm{H}) ; \mathrm{A}_{\mathrm{H}}\right.$ ), where $\mathrm{A}_{\mathrm{H}}$ is endowed with the conjugation action of $\pi_{0}(\mathrm{H})$ (see for example $[\mathbf{3}])$. Hence, to each $\pi_{0}(\mathrm{H})$-linear morphism $f: \mathrm{A}_{\mathrm{H}} \rightarrow G$, one associates the image of $[\mathrm{H}]$ by the induced morphism $\hat{f}: H^{3}\left(\pi_{0}(\mathrm{H}) ; \mathrm{A}_{\mathrm{H}}\right) \rightarrow H^{3}\left(\pi_{0}(\mathrm{H}) ; G\right)$.

Lemma 2.3.4. Suppose that the class $[\mathrm{H}]$ vanishes in $H^{3}\left(\pi_{0}(\mathrm{H}) ; \mathrm{A}_{\mathrm{H}}\right)$. Then for any commutative group $G$, there is a split exact sequence of abelian groups

$$
1 \rightarrow H^{2}\left(\pi_{0}(\mathrm{H}) ; G\right) \rightarrow \pi_{0}\left(\operatorname{Hom}_{\otimes}(\mathrm{H}, G[1])\right) \rightarrow \operatorname{Hom}_{\pi_{0}(\mathrm{H})}\left(\mathrm{A}_{\mathrm{H}}, G\right) \rightarrow 1 .
$$

Proof. By the above description of $\delta$, we clearly get the exact sequence (2.3.4). One possible way to show that it splits is the following. Since $[\mathrm{H}]$ is trivial in $H^{3}\left(\pi_{0}(\mathrm{H}) ; \mathrm{A}_{\mathrm{H}}\right)$, the "essentially exact" sequence of $g r$-categories

$$
1 \longrightarrow \mathrm{A}_{\mathrm{H}}[1] \longrightarrow \mathrm{H} \longrightarrow \pi_{0}(\mathrm{H})[0] \longrightarrow 1
$$

splits. This means that there is an equivalence of $g r$-categories $\mathrm{H} \simeq\left(\mathrm{A}_{\mathrm{H}} \stackrel{e}{\rightarrow} \pi_{0}(\mathrm{H})\right)$, where $e$ denotes the trivial homomorphism. Hence, a direct computation as in

\footnotetext{
${ }^{19}$ This is exactly the action described at the beginning of this section, since $\pi_{0}(\mathrm{H})=\mathrm{Pic}_{\mathrm{H}[1]}(\bullet)$ and $\mathrm{A}_{\mathrm{H}}=\mathrm{Z}_{\mathrm{H}[1]}(\bullet)$.

${ }^{20}$ If $\mathbf{H}=H^{-1} \stackrel{d}{\rightarrow} H^{0}$, this class coincides with the usual one in $H^{3}$ (coker $d$; ker $d$ ) attached to the underlying crossed module $H^{-1} \stackrel{d}{\rightarrow} H^{0}$. See for example [4, Chapter V].
} 
Lemma 2.3.3 shows that there is an isomorphism of groups

$$
\pi_{0}\left(\mathrm{Hom}_{\otimes}\left(\mathrm{A}_{\mathrm{H}} \stackrel{e}{\rightarrow} \pi_{0}(\mathrm{H}), G[1]\right)\right) \simeq H^{2}\left(\pi_{0}(\mathrm{H}) ; G\right) \times \operatorname{Hom}_{\pi_{0}(\mathrm{H})}\left(\mathrm{A}_{\mathrm{H}}, G\right) .
$$

For $\mathrm{H}=\Pi_{1}(\Omega X)$, we have $\pi_{0}\left(\Pi_{1}(\Omega X)\right)=\pi_{1}(X)$ and $\mathrm{A}_{\Pi_{1}(\Omega X)}=\pi_{2}(X)$ and the class $k_{2}(X)=\left[\Pi_{1}(\Omega X)\right]$ in $H^{3}\left(\pi_{1}(X) ; \pi_{2}(X)\right)$ is the so-called Postnikov $k$-invariant of $X$. Hence, using Lemma 2.3.3, 2.3.4 and Proposition 2.3.2, we get

Corollary 2.3.5 (Hopf's theorem for 2-cohomology). Let $X$ be connected and locally relatively 2-connected and $G$ a commutative group.

(i) There exists an exact sequence of abelian groups

$$
1 \rightarrow H^{2}\left(\pi_{1}(X) ; G\right) \rightarrow H^{2}\left(X ; G_{X}\right) \rightarrow \operatorname{Hom}_{\pi_{1}(X)}\left(\pi_{2}(X), G\right) \stackrel{\delta}{\rightarrow} H^{3}\left(\pi_{1}(X) ; G\right),
$$

where $G$ is view as a $\pi_{1}(X)$-module with trivial action.

(ii) If moreover the Postnikov k-invariant $k_{2}(X)$ vanishes in $H^{3}\left(\pi_{1}(X) ; \pi_{2}(X)\right)$, there is a split exact sequence of abelian groups

$$
1 \rightarrow H^{2}\left(\pi_{1}(X) ; G\right) \rightarrow H^{2}\left(X ; G_{X}\right) \rightarrow \operatorname{Hom}_{\pi_{1}(X)}\left(\pi_{2}(X), G\right) \rightarrow 1 .
$$

Recall that $H^{2}\left(\pi_{1}(X) ; G\right) \simeq \mathcal{E}_{c}\left(\pi_{1}(X) ; G\right)$, the group of equivalence classes of central extensions of $\pi_{1}(X)$ by $G$. Hence (2.3.5) takes a similar form as the Universal Coefficient Theorem. We refer to [4] for a classical proof of the homological version of Hopf's theorem.

Now, let $G$ be a not necessarily commutative group and consider $C=G[1]$. Then the 2-category $\boldsymbol{\Gamma}\left(X\right.$, End $\left.(G[1])[1]_{X}\right)$ is equivalent to the strict 2-category $\operatorname{Ger}\left(G_{X}\right)$ of $G_{X}$-gerbes, that is, stacks locally equivalent to $G[1]_{X}$. One may show (see for example $[\mathbf{3}]$ ) that there is an isomorphism of pointed sets

$$
\boldsymbol{\pi}_{0}\left(\operatorname{Ger}\left(G_{X}\right)\right) \simeq H^{1}\left(X ; G_{X} \stackrel{\text { ad }}{\longrightarrow} \operatorname{Aut}_{\mathrm{Gr}}\left(G_{X}\right)\right),
$$

where the right hand side is the first cohomology set of $X$ with values in the sheaf of crossed modules $G_{X} \stackrel{\text { ad }}{\longrightarrow} \operatorname{Aut}_{\mathrm{Gr}}\left(G_{X}\right)$.

Assume that $X$ is locally relatively 2-connected. By Theorem 2.2.5, there is an equivalence of 2-categories

$$
\operatorname{Ger}\left(G_{X}\right) \stackrel{\sim}{\longrightarrow} \operatorname{Hom}\left(\Pi_{2}(X), \text { End }(G[1])[1]\right) .
$$

Since $\operatorname{Pic}(G[1]) \simeq \operatorname{Out}_{G r}(G)$, from $(2.3 .2)$ we get

Proposition 2.3.6 (Hurewicz-Hopf's formula II). Let $X$ be connected and locally relatively 2-connected. Then for any group $G$ there is an isomorphism of pointed sets

$$
H^{1}\left(X ; G_{X} \stackrel{\text { ad }}{\longrightarrow} \operatorname{Aut}_{\mathrm{Gr}}\left(G_{X}\right)\right) \stackrel{\sim}{\longrightarrow} \pi_{0}\left(\operatorname{Hom}_{\otimes}\left(\Pi_{1}(\Omega X), G \stackrel{\text { ad }}{\longrightarrow} \operatorname{Aut}_{\mathrm{Gr}}(G)\right)\right) / \operatorname{Out}_{\mathrm{Gr}}(G) .
$$

With similar computations as for the commutative case, we get the following 
Lemma 2.3.7. Let $\mathrm{H}$ be a gr-category and $\mathrm{G}=G \stackrel{\text { ad }}{\longrightarrow} \operatorname{Aut}_{\mathrm{Gr}}(G)$, for a group $G$. Then (2.3.3) gives an exact sequence of pointed sets

$$
1 \rightarrow \mathcal{E}\left(\pi_{0}(\mathrm{H}) ; G\right) \rightarrow \pi_{0}\left(\mathrm{Hom}_{\otimes}\left(\mathrm{H}, G \stackrel{\text { ad }}{\longrightarrow} \operatorname{Aut}_{\mathrm{Gr}}(G)\right)\right) \rightarrow \operatorname{Hom}_{\mathrm{Gr}}\left(\mathrm{A}_{\mathrm{H}}, \operatorname{ker} d\right),
$$

where the first term is the set of equivalence classes of extensions of $\pi_{0}(\mathrm{H})$ by $G$.

For a group $H$, denote by $H^{1}\left(H ; G \stackrel{\text { ad }}{\longrightarrow} \operatorname{Aut}_{\mathrm{Gr}}(G)\right)$ the first cohomology set of $H$ with values in the crossed module $G \stackrel{\text { ad }}{\longrightarrow} \operatorname{Aut}_{G r}(G)$ (see for example [2]). By definition, there is a pointed set isomorphism $H^{1}\left(H ; G \stackrel{\text { ad }}{\longrightarrow} \operatorname{Aut}_{\mathrm{Gr}}(G)\right) \simeq$ $\mathcal{E}(H ; G) / \operatorname{Out}_{\mathrm{Gr}}(G)$, where the action of $\operatorname{Out}_{\mathrm{Gr}}(G)$ is by conjugation. Hence, combining Proposition 2.3.6 and Lemma 2.3.7 with $\mathrm{H}=\Pi_{1}(\Omega X)$, we get

Corollary 2.3.8 (Hopf's theorem for non abelian 2-cohomology). Let $X$ be connected and locally relatively 2-connected. Then for each group $G$ there is an exact sequence of pointed sets

$$
\begin{aligned}
1 \longrightarrow H^{1}\left(\pi_{1}(X) ; G \stackrel{\text { ad }}{\longrightarrow} \operatorname{Aut}_{\mathrm{Gr}}(G)\right) \longrightarrow & H^{1}\left(X ; G_{X} \stackrel{\text { ad }}{\longrightarrow} \operatorname{Aut}_{\mathrm{Gr}}\left(G_{X}\right)\right) \longrightarrow \\
& \longrightarrow \operatorname{Hom}_{\mathrm{Gr}}\left(\pi_{2}(X), \mathrm{Z}(G)\right) / \operatorname{Out}_{\mathrm{Gr}}(G),
\end{aligned}
$$

where $\operatorname{Out}_{\mathrm{Gr}}(G)$ acts on the left on $\mathrm{Hom}_{\mathrm{Gr}}\left(\pi_{2}(X), \mathrm{Z}(G)\right)$ by composition. If moreover $\pi_{1}(X)$ is trivial, one gets an isomorphism ("Hurewicz's formula")

$$
H^{1}\left(X ; G_{X} \stackrel{\text { ad }^{\longrightarrow}}{\longrightarrow} \operatorname{Aut}_{\mathrm{Gr}}\left(G_{X}\right)\right) \stackrel{\sim}{\longrightarrow} \operatorname{Hom}_{\mathrm{Gr}}\left(\pi_{2}(X), \mathrm{Z}(G)\right) / \operatorname{Out}_{\mathrm{Gr}}(G),
$$

A similar result holds replacing $G \stackrel{\text { ad }}{\longrightarrow} \operatorname{Aut}_{\mathrm{Gr}}(G)$ by a general crossed module $G^{-1} \stackrel{d}{\rightarrow}$ $G^{0}$.

\section{Final comments}

What's next? It seems clear that, using the same techniques, one should expect for each $n$-category $\mathbf{C}$ and each locally relatively $n$-connected space $X$ a natural $n$-equivalence

$$
\boldsymbol{\mu}^{\boldsymbol{n}}: \boldsymbol{\Gamma}\left(X, \mathbf{C}_{X}\right) \stackrel{\sim}{\longrightarrow} \mathbf{H o m}_{n \mathbf{C a t}}\left(\Pi_{n}(X), \mathbf{C}\right),
$$

where $\boldsymbol{\Gamma}\left(X, \mathbf{C}_{X}\right)$ denotes the global sections of the constant $n$-stack with stalk $\mathbf{C}$, $\Pi_{n}(X)$ the homotopy $n$-groupoid of $X$ and $\mathbf{H o m}_{n \mathbf{C a t}}(\cdot, \cdot)$ the $n$-category of $n$ functors. However, some care has to be taken since there are several non-equivalent definitions of $n$-categories for $n \geqslant 3$ (see for example [11]). We will not investigate this problem any further here. An answer in this direction, using the formalism of Segal categories, is partially given in $[\mathbf{1 3}]$ for $\mathbf{C}=(n-1) \mathbf{C a t}$, the strict $n$-category of $(n-1)$-categories, and $X$ a pointed and connected $C W$-complex.

Let us suppose for a while that formula (2.3.6) is valid and consider a commutative group $G$. Denote by $G[n]$ the strict $g r$ - $n$-category with a single element, trivial 
$i$-arrows for $i \leqslant n-1$ and $G$ as $n$-arrows. Then one may check that there is an isomorphism of groups

$$
H^{n}\left(X ; G_{X}\right) \simeq \boldsymbol{\pi}_{0}\left(\boldsymbol{\Gamma}\left(X, G[n]_{X}\right)\right),
$$

where the right-hand side is the group of $n$-equivalence classes of global objects in $G[n]_{X}$

Set $H^{n}\left(\Pi_{n}(X) ; G\right)=\boldsymbol{\pi}_{0}\left(\mathbf{H o m}_{n \mathbf{C a t}}\left(\Pi_{n}(X), G[n]\right)\right)$. Then, if $X$ is locally relatively $n$-connected, from (2.3.6), we have an isomorphism of groups

$$
H^{n}\left(X ; G_{X}\right) \simeq H^{n}\left(\Pi_{n}(X) ; G\right) .
$$

This isomorphism should be interpreted as the "Hurewicz-Hopf's formula". Indeed, if we suppose that $X$ is connected and that $\pi_{i}(X) \simeq 1$ for all $2 \leqslant i \leqslant n-1$, we get an "essentially exact" sequence of $g r-(n-1)$-categories

$$
1 \longrightarrow \pi_{n}(X)[n-1] \longrightarrow \Pi_{n-1}(\Omega X) \longrightarrow \pi_{1}(X)[0] \longrightarrow 1,
$$

and hence an exact sequence of groups

$1 \longrightarrow \boldsymbol{\pi}_{0}\left(\mathbf{H o m}_{\otimes}\left(\pi_{1}(X)[0], G[n-1]\right)\right) \longrightarrow \boldsymbol{\pi}_{0}\left(\mathbf{H o m}_{\otimes}\left(\Pi_{n-1}(\Omega X), G[n-1]\right)\right) \longrightarrow$

$$
\longrightarrow \boldsymbol{\pi}_{0}\left(\mathbf{H o m}_{\otimes}\left(\pi_{n}(X)[n-1], G[n-1]\right)\right)
$$

From the isomorphism (2.3.7) and a direct calculation, we shall finally get the Hopf's exact sequence

$$
1 \rightarrow H^{n}\left(\pi_{1}(X) ; G\right) \rightarrow H^{n}\left(X ; G_{X}\right) \rightarrow \operatorname{Hom}_{\mathrm{Gr}}\left(\pi_{n}(X), G\right),
$$

where $G$ is view as a $\pi_{1}(X)$-module with trivial action. If moreover $\pi_{1}(X) \simeq 1$, the Hurewicz morphism $H^{n}\left(X ; G_{X}\right) \rightarrow \operatorname{Hom}_{\mathrm{Gr}}\left(\pi_{n}(X), G\right)$ is an isomorphism. We refer to $[8]$ for a classical proof of this result.

If $G$ is a not necessarily commutative group, we define the $n$-groupoid $G \llbracket n \rrbracket$ by induction as

$$
G \llbracket 1 \rrbracket=G[1], \quad G \llbracket n+1 \rrbracket=\mathbf{A u t}_{n \mathbf{C a t}}(G \llbracket n \rrbracket)[1],
$$

where $\mathbf{A u t}_{n \mathbf{C a t}}(G \llbracket n \rrbracket)$ denotes the $g r$ - $n$-category of auto- $n$-equivalence of $G \llbracket n \rrbracket$. Note that, when $G$ is commutative, if we require $G$-linearity at each step in the definition of $G \llbracket n \rrbracket$, we recover $G[n]$. Then one may define the non abelian $n$-cohomology set of $X$ with coefficients in $G_{X}$ as

$$
H_{g}^{n}\left(X ; G_{X}\right)=\boldsymbol{\pi}_{0}\left(\boldsymbol{\Gamma}\left(X, G \llbracket n \rrbracket_{X}\right)\right),
$$

and the non abelian $n$-cohomology set of $\Pi_{n}(X)$ with coefficients in $G$ as

$$
H_{g}^{n}\left(\Pi_{n}(X) ; G\right)=\boldsymbol{\pi}_{0}\left(\mathbf{H o m}_{n \mathbf{C a t}}\left(\Pi_{n}(X), G \llbracket n \rrbracket\right)\right) .
$$

Note that, if $n=2$ there is an isomorphism $H_{g}^{2}\left(X ; G_{X}\right) \simeq H^{1}\left(X ; G_{X} \stackrel{\text { ad }}{\longrightarrow}\right.$ Aut $\left._{\mathrm{Gr}}\left(G_{X}\right)\right)$, but $H_{g}^{2}\left(\Pi_{2}(X) ; G\right)$ is not isomorphic to $H^{1}\left(\pi_{1}(X) ; G \stackrel{\text { ad }}{\longrightarrow} \operatorname{Aut}_{\mathrm{Gr}}(G)\right)$ in general. 
If $X$ is locally relatively $n$-connected, then the $n$-equivalence (2.3.6) gives an isomorphism of pointed sets

$$
H_{g}^{n}\left(X ; G_{X}\right) \simeq H_{g}^{n}\left(\Pi_{n}(X) ; G\right) .
$$

This is the very non abelian version of the "Hurewicz-Hopf's formula".

\section{A. The stack of sheaves with values in a complete category}

We recall here the construction of the stack of sheaves with values in a complete category C, i.e. a category which admits all small limits. (See Appendix B for the definition of a stack.)

Let $X$ be a topological space and denote by $\operatorname{Op}(X)$ the category of its open subset with inclusions morphisms.

Definition A.0.9. A presheaf on $X$ with values in $\mathrm{C}$ is a functor

$$
\mathrm{Op}(X)^{\mathrm{op}} \longrightarrow \mathrm{C} \text {. }
$$

A morphism between presheaves is a morphism of functors. We denote by $\mathrm{PSh}_{X}(\mathrm{C})$ the category of presheaves on $X$ with values in C.

A presheaf is called a sheaf if it commutes to filtered limits indexed by coverings that are stable by finite intersections, and we denote by $\mathrm{Sh}_{X}(\mathrm{C})$ the full subcategory of $\mathrm{PSh}_{X}(\mathrm{C})$ whose objects are sheaves ${ }^{21}$.

Note that if $U \subset X$ is an open subset and $\mathcal{F}$ is a sheaf on $X$, then its restriction $\left.\mathcal{F}\right|_{U}$ is also a sheaf. Hence we can define the prestack of sheaves on $X$, denoted by $\mathfrak{S h}_{X}(\mathrm{C})$, by assigning $X \supset U \mapsto \mathrm{Sh}_{U}(\mathrm{C})$.

Let $\mathcal{F}, \mathcal{G}$ be two presheaves on $X$. We have a natural bijective map of sets

$$
\operatorname{Hom}_{\mathrm{PSh}_{X}(\mathrm{C})}(\mathcal{F}, \mathcal{G}) \stackrel{\sim}{\longrightarrow} \lim _{\substack{(U, V) \\ V \subset U}} \operatorname{Hom}_{\mathrm{C}}(\mathcal{F}(U), \mathcal{G}(V))
$$

where $(U, V)$ is considered as an object of $\mathrm{Op}(X)^{\mathrm{op}} \times \mathrm{Op}(X)$. Now let $U \subset X$ be an open subset, $\mathcal{F}$ a presheaf on $X$ and $\mathcal{G}$ a presheaf on $U$. Then it is easy to see that we have the isomorphism of sets

$$
\begin{aligned}
& \operatorname{Hom}_{\mathrm{PSh}_{U}(\mathrm{C})}\left(\left.\mathcal{F}\right|_{U}, \mathcal{G}\right) \stackrel{\sim}{\longrightarrow} \lim _{\substack{(V, W) \\
W \subset V \subset U}} \operatorname{Hom}_{\mathrm{C}}(\mathcal{F}(V), \mathcal{G}(W)) \\
& \stackrel{\sim}{\longrightarrow} \lim _{\substack{V, W \\
W \subset V \subset X}} \operatorname{Hom}_{\mathrm{C}}(\mathcal{F}(V), \mathcal{G}(W \cap U))
\end{aligned}
$$

\footnotetext{
${ }^{21}$ Recall that, if $\mathcal{F}$ is a presheaf of sets the sheaf condition means that for any open subset $U \subset X$, and any open covering $\left\{U_{i}\right\}_{i \in I}$ of $U$, the natural sequence given by the restriction maps

$$
\mathcal{F}(U) \longrightarrow \prod_{i \in I} \mathcal{F}\left(U_{i}\right) \Longrightarrow \prod_{i, j \in I} \mathcal{F}\left(U_{i j}\right)
$$
}

is exact in the usual sense. 
Lemma A.0.10. Let $\mathcal{F}$ be a presheaf and $\mathcal{G}$ a sheaf on $X$. Then the presheaf $\mathcal{H} m_{\mathrm{PSh}_{X}(\mathrm{C})}(\mathcal{F}, \mathcal{G})$ defined by

$$
X \supset U \mapsto \mathcal{H} \operatorname{mom}_{\mathrm{PSh}_{X}(\mathrm{C})}(\mathcal{F}, \mathcal{G})(U)=\operatorname{Hom}_{\mathrm{PSh}_{U}(\mathrm{C})}\left(\left.\mathcal{F}\right|_{U},\left.\mathcal{G}\right|_{U}\right)
$$

is a sheaf of sets.

Proof. We have to show that $\mathcal{H o m}_{\mathrm{PSh}_{X}(\mathrm{C})}(\mathcal{F}, \mathcal{G})$ commutes to small filtered limits indexed by coverings that are stable by finite intersection. Let $\left\{U_{i}\right\}_{i \in I}$ be such a covering of an open subset $U \subset X$. Then we have

$$
\begin{aligned}
& \mathcal{H} \operatorname{mom}_{\mathrm{PSh}_{X}(\mathrm{C})}(\mathcal{F}, \mathcal{G})(U)=\operatorname{Hom}_{\mathrm{PSh}_{U}(\mathrm{C})}\left(\left.\mathcal{F}\right|_{U},\left.\mathcal{G}\right|_{U}\right) \\
& \simeq \lim _{\substack{(\overleftarrow{W}, W \\
W \subset V}} \operatorname{Hom}_{\mathrm{C}}(\mathcal{F}(V), \mathcal{G}(U \cap W)) \\
& \simeq \lim _{\substack{(W, W) \\
W \subset V}} \operatorname{Hom}_{\mathrm{C}}\left(\mathcal{F}(V),{\underset{\lim }{i \in I}}_{\mathfrak{Z}} \mathcal{G}\left(U_{i} \cap W\right)\right) \\
& \simeq \lim _{\substack{(V, W) \\
W \subset V}}{\underset{i m}{i \in I}}_{\overleftarrow{C}} \operatorname{Hom}_{\mathrm{C}}\left(\mathcal{F}(V), \mathcal{G}\left(U_{i} \cap W\right)\right) \\
& \simeq \lim _{i \in I} \lim _{\substack{V, W \\
W \subset V}} \operatorname{Hom}_{\mathrm{C}}\left(\mathcal{F}(V), \mathcal{G}\left(U_{i} \cap W\right)\right) \\
& \simeq \lim _{i \in I} \operatorname{Hom}_{\mathrm{PSh}_{U_{i}}(\mathrm{C})}\left(\left.\mathcal{F}\right|_{U_{i}},\left.\mathcal{G}\right|_{U_{i}}\right) \\
& \simeq \lim _{i \in I} \mathcal{H} \operatorname{Hom}_{\mathrm{PSh}_{X}(\mathrm{C})}(\mathcal{F}, \mathcal{G})\left(U_{i}\right)
\end{aligned}
$$

Lemma A.0.11. Let $\mathcal{F}$ be a presheaf on $X$. Then $\mathcal{F}$ is a sheaf if and only if for any object $A \in \mathrm{ObC}$ and any open subset $U \subset X$ the presheaf

$$
U \supset V \mapsto \operatorname{Hom}_{\mathrm{C}}(A, \mathcal{F}(V))
$$

is a sheaf of sets.

Proof. Follows immediately from Yoneda's Lemma.

Proposition A.0.12. The prestack $\mathfrak{S h}_{X}(\mathrm{C})$ of sheaves with values in $\mathrm{C}$ is a stack.

Proof. By Lemma A.0.10, the prestack is separated. Now let $\left(\left\{U_{i}\right\}_{i \in I},\left\{\mathcal{F}_{i}\right\}_{i \in I},\left\{\theta_{i j}\right\}_{i, j \in I}\right)$ be a descent datum for $\mathfrak{S h}_{X}(\mathrm{C})$ on open subset $U \subset X$. By taking a refinement, we can assume that the covering $\left\{U_{i}\right\}_{i \in I}$ is stable by finite intersections.

Let $V \subset U$. Then the cocycle condition allows us to define

$$
\mathcal{F}(V)=\lim _{i \in I} \mathcal{F}_{i}\left(V \cap U_{i}\right)
$$

It is then obvious that $\mathcal{F}$ is a sheaf (for instance using Lemma A.0.11 and the fact that this is true if $\mathrm{C}=$ Set) which by construction is isomorphic to $\mathcal{F}_{i}$ on $U_{i}$ by an isomorphism $\theta_{i}$ such that $\theta_{i j} \circ \theta_{j}=\theta_{i}$ on $U_{i} \cap U_{j}$. 
Proposition A.0.13. The stack $\mathfrak{S h}_{X}(\mathrm{C})$ admits all small limits.

Proof. Let $\beta: \mathrm{I} \rightarrow \mathrm{Sh}_{X}(\mathrm{C})$ be a functor, with I a small category. Then, for each open subset $U \subset X$, set

$$
\mathcal{F}(U)=\lim _{i \in \mathrm{I}} \beta(i)(U)
$$

It is immediately verified that $\mathcal{F}$ is a sheaf on $X$ that satisfies $\mathcal{F} \simeq \lim _{i \in 1} \beta(i)$.

Definition A.0.14. Let $\mathcal{F}$ be a presheaf. A sheaf $\widetilde{\mathcal{F}}$ together with a morphism $\mathcal{F} \rightarrow \widetilde{\mathcal{F}}$ is called the sheaf associated to $\mathcal{F}$ if it satisfies the usual universal property, i.e. any morphism from $\mathcal{F}$ into a sheaf $\mathcal{G}$ factors uniquely through $\widetilde{\mathcal{F}}$ :

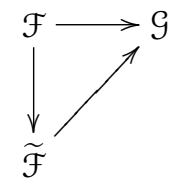

In general, there may not exist a functor which assigns to each presheaf its associated sheaf. However, we have

Proposition A.0.15. Assume that $X$ is locally connected. Then for each $M \in \mathrm{ObC}$ the sheaf associated to the constant presheaf with stalk $M$ exists.

Proof. Let $U \subset X$ be an open subset. Denote by \#U the set of connected components of $U$ and set

$$
M_{X}(U)=M^{\# U} .
$$

Let $x \in U$. We denote by $\bar{x}_{U}$ its class in \#U. Then for any inclusion $V \subset U$ of open subsets and for any $x \in V$, the natural morphisms

$$
M_{X}(U) \rightarrow M_{\bar{x}_{U}} \rightarrow M_{\bar{x}_{V}}
$$

define the restriction morphism

$$
M_{X}(U) \rightarrow M_{X}(V) .
$$

Since we know that this is a sheaf if $\mathrm{C}=$ Set, Lemma A.0.11 implies that $M_{X}$ is a sheaf which verifies the desired universal property.

Definition A.0.16. Let $M \in \mathrm{ObC}$. The sheaf associated to the constant presheaf with stalk $M$ is called the constant sheaf with stalk $M$, and we denote it by $M_{X}$.

Remark A.0.17. The hypothesis on the connectivity of $X$ in the Proposition A.0.15 is necessary to recover the classical definition of constant sheaf. More precisely, if $M$ is a set and $M_{X}$ is the constant sheaf defined in the usual way, there is a natural injective map (the 0-monodromy) $\mu^{0}: M_{X}(X) \rightarrow \operatorname{Hom}(\# X, M) \simeq M^{\# X}$ defined by $\mu^{0}(s)\left(\bar{x}_{X}\right)=s\left(\bar{x}_{X}\right)$ for a section $s$ in $M_{X}(X)$. Clearly, if $X$ is locally connected $\mu^{0}$ is a bijection. 
Let $X$ be a locally connected topological space. Denote by $\mathrm{CSh}_{X}(\mathrm{C})$ the full subcategory of $\mathrm{Sh}_{X}(\mathrm{C})$ of constant sheaves. The previous construction defines a faithful functor

$$
(\cdot)_{X}: \mathrm{C} \longrightarrow \mathrm{CSh}_{X}(\mathrm{C}),
$$

which is an equivalence if $X$ is connected (a quasi-inverse is given by the global sections functor).

Definition A.0.18. A sheaf $\mathcal{F}$ is called locally constant if there is an open covering $X=\bigcup U_{i}$ such that $\left.\mathcal{F}\right|_{U_{i}}$ is isomorphic to a constant sheaf.

We denote by $\mathfrak{L}_{\mathfrak{c}} \mathfrak{h h}_{X}(\mathrm{C})$ the full substack of $\mathfrak{S h}_{X}(\mathrm{C})$ whose objects are the locally constant sheaves.

\section{B. The 2-stack of stacks with values in a 2-complete 2-category}

Let us recall the construction of the 2 -stack of stacks with values in a 2-complete 2-category $\mathbf{C}$, i.e. a 2-category which admits all small 2-limits. (References for the basic definitions about 2-stacks are made to $[\mathbf{2}]$.) Recall that the 2-Yoneda lemma states that the 2 -functor

$$
\mathbf{C} \longrightarrow \widehat{\mathbf{C}}=\operatorname{Hom}\left(\mathbf{C}^{\text {op }}, \mathbf{C a t}\right), \quad \mathrm{P} \mapsto \operatorname{Hom}_{\mathbf{C}}(\cdot, \mathrm{P})
$$

is fully faithful (see for example [11, Chapter 1] for more details). Since $\widehat{\mathbf{C}}$ is strict, the reader may assume for sake of simplicity that $\mathbf{C}$ is a strict 2-category.

Let $X$ be a topological space and denote by $\mathbf{O p}(X)$ the 2-category of its open subsets, obtained by trivially enriching $\mathrm{Op}(X)$ with identity 2-arrows.

Definition B.0.19. A prestack on $X$ with values in $\mathbf{C}$ is a 2 -functor

$$
\mathrm{Op}(X)^{\mathrm{op}} \longrightarrow \mathbf{C} \text {. }
$$

A functor between prestacks is a 2-transformation of 2-functors and transformations of functors of prestacks are modifications of 2-transformations of 2-functors. We denote by $\mathbf{P S t}_{X}(\mathbf{C})$ the 2-category of prestacks on $X$ with values in $\mathbf{C}$.

A prestack is called a stack if it commutes to filtered 2-limits indexed by coverings that are stable by finite intersections, and we denote by $\mathbf{S t}_{X}(\mathbf{C})$ the full subcategory of $\mathbf{P S t}_{X}(\mathbf{C})$ whose objects are stacks ${ }^{22}$.

If $\mathfrak{S}$ is a stack of categories, then for any objects $P, Q \in \mathfrak{S}(U)$ on some open subset $U \subset X$, the presheaf $U \supset V \mapsto \operatorname{Hom}_{\mathfrak{S}(V)}\left(\left.P\right|_{V},\left.Q\right|_{V}\right)$ is a sheaf of sets on $U$. We say that a prestack of categories is separated if it has this property.

Note that if $U \subset X$ is an open subset and $\mathfrak{S}$ is a stack on $X$, then its restriction $\left.\mathfrak{S}\right|_{U}$ is also a stack. Hence the assignment $X \supset U \mapsto \mathbf{S t}_{U}(\mathbf{C})$ defines the pre-2-stack

${ }^{22}$ Similarly to the definition of sheaf, if $\mathfrak{S}$ is a prestack of categories the stack condition means that for any open subset $U \subset X$, and any open covering $\left\{U_{i}\right\}_{i \in I}$ of $U$, the natural sequence given by the restriction functors

$$
\mathfrak{S}(U) \longrightarrow \prod_{i \in I} \mathfrak{S}\left(U_{i}\right) \Longrightarrow \prod_{i, j \in I} \mathfrak{S}\left(U_{i j}\right) \Longrightarrow \prod_{i, j, k \in I} \mathfrak{S}\left(U_{i j k}\right)
$$

is exact in the sense of [SGA1, exposé XIII]. 
of stacks on $X$ with values in $\mathbf{C}$, which we denote by $\mathfrak{S t}_{X}(\mathbf{C})$.

Let $\mathfrak{S}, \mathfrak{T}$ be two prestacks on $X$. We have a natural equivalence of categories

$$
\operatorname{Hom}_{\mathbf{P S t}_{X}(\mathbf{C})}(\mathfrak{S}, \mathfrak{T}) \stackrel{\sim}{\longrightarrow} \lim _{\substack{(U, V) \\ V \subset U}} \operatorname{Hom}_{\mathbf{C}}(\mathfrak{S}(U), \mathfrak{T}(V))
$$

where $(U, V)$ is considered as an object of $\mathbf{O p}(X)^{\mathrm{op}} \times \mathbf{O} \mathbf{p}(X)$. Now let $U \subset X$ be an open subset, $\mathfrak{S}$ a prestack on $X$ and $\mathfrak{T}$ a prestack on $U$. Then it is easy to see that we have the equivalence of categories

$$
\begin{aligned}
\operatorname{Hom}_{\mathbf{P S t}_{U}(\mathbf{C})}\left(\left.\mathfrak{S}\right|_{U}, \mathfrak{T}\right) & \stackrel{\sim}{\longrightarrow} \lim _{\substack{(V, W) \\
W \subset V \subset U}} \operatorname{Hom}_{\mathbf{C}}(\mathfrak{S}(V), \mathfrak{T}(W)) \\
& \stackrel{\sim}{\longrightarrow} \lim _{\substack{(\overleftarrow{W} W) \\
W \subset V \subset X}} \operatorname{Hom}_{\mathbf{C}}(\mathfrak{S}(V), \mathfrak{T}(W \cap U)) .
\end{aligned}
$$

Hence, we have

Lemma B.0.20. Let $\mathfrak{S}$ be a prestack and $\mathfrak{T}$ be a stack on $X$.

Then the prestack $\mathfrak{H o m}_{\mathbf{P S t}_{X}(\mathbf{C})}(\mathfrak{S}, \mathfrak{T})$ defined by

$$
\mathfrak{H o m}_{\mathbf{P S t}_{X}(\mathbf{C})}(\mathfrak{S}, \mathfrak{T})(U)=\operatorname{Hom}_{\mathbf{P S t}_{U}(\mathbf{C})}\left(\left.\mathfrak{S}\right|_{U},\left.\mathfrak{T}\right|_{U}\right)
$$

is a stack of categories.

Moreover, using the 2-Yoneda's Lemma, we get

Lemma B.0.21. Let $\mathfrak{S}$ be a prestack on $X$. Then $\mathfrak{S}$ is a stack if and only if for any object $\mathrm{P} \in \mathrm{Ob} \mathbf{C}$ and any open subset $U \subset X$ the prestack

$$
U \supset V \mapsto \operatorname{Hom}_{\mathbf{C}}(\mathrm{P}, \mathfrak{S}(V))
$$

is a stack of categories.

Proposition B.0.22. The pre-2-stack $\mathbf{S t}_{X}(\mathbf{C})$ of stacks with values in $\mathbf{C}$ is a 2-stack.

Proof. By Lemma B.0.20, the pre-2-stack is separated. Now let

$$
\left(\left\{U_{i}\right\}_{i \in I},\left\{\mathfrak{S}_{i}\right\}_{i \in I},\left\{F_{i j}\right\}_{i, j \in I},\left\{\varphi_{i j k}\right\}_{i, j, k \in I}\right)
$$

be a descent datum for $\mathfrak{S t}_{X}(\mathbf{C})$ on open subset $U \subset X$. This means that $\left\{U_{i}\right\}_{i \in I}$ is an open covering of $U, \mathfrak{S}_{i}$ are stacks on $U_{i}, F_{i j}:\left.\left.\mathfrak{S}_{j}\right|_{U_{i j}} \stackrel{\sim}{\longrightarrow} \mathfrak{S}_{i}\right|_{U_{i j}}$ are equivalences of stacks and $\varphi_{i j k}: \varphi_{i j} \circ \varphi_{j k} \rightarrow \varphi_{i k}$ are invertible transformations of functors from $\left.\mathfrak{S}_{k}\right|_{U_{i j k}}$ to $\left.\mathfrak{S}_{i}\right|_{U_{i j k}}$, such that for any $i, j, k, l \in I$, the following diagram of transformations of functors from $\left.\mathfrak{S}_{l}\right|_{U_{i j k l}}$ to $\left.\mathfrak{S}_{i}\right|_{U_{i j k l}}$ commutes

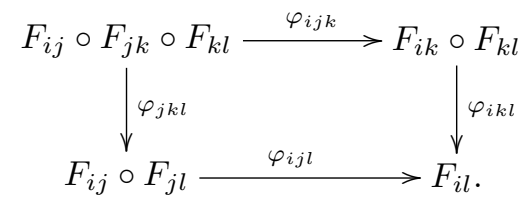

By taking a refinement, we can assume that the covering $\left\{U_{i}\right\}_{i \in I}$ is stable by finite intersections. 
Let $V \subset U$ be an open subset. Then the cocycle condition (B.0.8) allows us to define the category

$$
\mathfrak{S}(V)=2 \lim _{i \in I} \mathfrak{S}_{i}\left(V \cap U_{i}\right)
$$

It is then obvious that the assignment $U \supset V \mapsto \mathfrak{S}(V)$ defines a stack $\mathfrak{S}$ (for instance using Lemma B.0.21 and the fact that this is true if $\mathbf{C}=\mathbf{C a t}$ ) and that, by construction, there are e quivalences of stacks $F_{i}:\left.\mathfrak{S}\right|_{U_{i}} \stackrel{\sim}{\longrightarrow} \mathfrak{S}_{i}$. Moreover, one checks that there exist invertible transformations of functors $\varphi_{i j}:\left.F_{i j} \circ F_{j}\right|_{U_{i j}} \stackrel{\sim}{\longrightarrow}$ $\left.F_{i}\right|_{U_{i j}}$ such that $\left.\left.\varphi_{i j}\right|_{U_{i j k}} \circ \varphi_{j k}\right|_{U_{i j k}}=\left.\varphi_{i k}\right|_{U_{i j k}} \circ \varphi_{i j k}$.

Proposition B.0.23. The 2-stack $\mathfrak{S t}_{X}(\mathbf{C})$ admits all small 2-limits.

Proof. Let $\beta: \mathbf{I} \rightarrow \mathbf{S t}_{X}(\mathbf{C})$ be a 2-functor, with $\mathbf{I}$ a small 2-category. Then, for each open subset $U \subset X$, set

$$
\mathfrak{S}(U)=2 \lim _{i \in \mathbf{I}} \beta(i)(U)
$$

It is immediately verified that $\mathfrak{S}$ is a stack on $X$ that satisfies $\mathfrak{S} \simeq \underset{i \in \mathbf{I}}{2 \lim _{i}} \beta(i)$.

Definition B.0.24. Let $\mathfrak{S}$ be a prestack. A stack $\widetilde{\mathfrak{S}}$ together with a functor $\mathfrak{S} \longrightarrow$ $\widetilde{\mathfrak{S}}$ is called the stack associated to $\mathfrak{S}$ if it satisfies the usual universal property, i.e. any functor from $\mathfrak{S}$ into a stack $\mathfrak{T}$ factors through $\widetilde{\mathfrak{S}}$ up to unique equivalence:

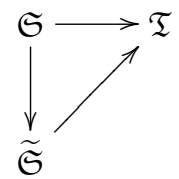

As for presheaves, in general there may not exist a stack associated to a given prestack. But we still have

Proposition B.0.25. Assume that $X$ is locally relatively 1-connected. Then for any $\mathrm{P} \in \mathrm{Ob} \mathbf{C}$, the stack associated to the constant prestack with stalk $\mathrm{P}$ exists.

Proof. Let $U \subset X$ be an open subset. Set

$$
\mathrm{P}_{X}(U)=\mathrm{P}^{\Pi_{1}(U)},
$$

where $\mathrm{P}^{\Pi_{1}(U)}$ denotes the 2-limit of the constant 2-functor $\Delta(P): \Pi_{1}(U) \longrightarrow \mathbf{C}$ at P. Let $x \in V \subset U$ and denote by $x_{U}$ the image of $x$ in $\Pi_{1}(U)$ by the natural functor $\Pi_{1}(V) \longrightarrow \Pi_{1}(U)$. Set $\mathrm{P}_{x_{U}}=\Delta(P)\left(x_{U}\right)$ and similarly for $\mathrm{P}_{x_{V}}$. Then we have the natural 1-arrows in $\mathbf{C}$

$$
\mathrm{P}_{X}(U) \longrightarrow \mathrm{P}_{x_{U}} \longrightarrow \mathrm{P}_{x_{V}}
$$

which define the 1-arrow

$$
\mathrm{P}_{X}(U) \longrightarrow \mathrm{P}_{X}(V)
$$

If $\mathbf{C}=\mathbf{C a t}$, then $\mathrm{P}=\mathrm{C}$ is a category and by Theorem 1.2 .9 there are equivalences $\mathrm{C}^{\Pi_{1}(U)} \simeq \operatorname{Hom}\left(\Pi_{1}(U), \mathrm{C}\right) \underset{\mu}{\stackrel{\sim}{\sim}} \mathrm{C}_{U}(U)$, hence the assignment $X \supset U \mapsto \mathrm{C}^{\Pi_{1}(U)}$ 
defines the stack of locally constant sheaves on $U$ with values in C. In the general case, one use Lemma B.0.21 to show that this construction gives a stack which verifies the desired universal property.

Definition B.0.26. Let $\mathrm{P} \in \mathrm{Ob} \mathbf{C}$. The stack associated to the constant prestack with stalk $\mathrm{P}$ is called the constant stack with stalk $\mathrm{P}$, and we denote it by $\mathrm{P}_{X}$.

Let $X$ be a locally relatively 1-connected topological space. Denote by $\mathbf{C S t}_{X}(\mathbf{C})$ the full sub-2-category of $\mathbf{S t}_{X}(\mathbf{C})$ of constant stacks. The previous construction defines a faithful 2-functor

$$
(\cdot)_{X}: \mathbf{C} \longrightarrow \mathbf{C S t}_{X}(\mathbf{C}),
$$

which is an equivalence if $X$ is 1-connected (a quasi-2-inverse is given by the global sections 2-functor). This easily follows from Corollary 1.2.8.

Definition B.0.27. A stack $\mathfrak{S}$ is called locally constant if there exists an open covering $X=\bigcup U_{i}$ such that $\left.\mathfrak{S}\right|_{U_{i}}$ is isomorphic to a constant stack.

We denote by $\mathfrak{L c S h}_{X}(\mathbf{C})$ the full sub-2-stack of $\mathfrak{S t}_{X}(\mathbf{C})$ whose objects are the locally constant stacks. 


\section{References}

[1] F. Borceux, Handbook of categorical algebra. 1. Basic category theory, Encyclopedia of Mathematics and its Applications, 50, Cambridge University Press, Cambridge (1994).

[2] L. Breen, Bitorseurs et cohomologie non abélienne, The Grothendieck Festschrift, Vol. I, Birkhäuser Boston (1990), 401-476.

[3] - On the classification of 2-gerbes and 2-stacks, Astérisque (1994), no. 225 .

[4] K.S. Brown, Cohomology of groups, corrected reprint of the 1982 original, Graduate Texts in Mathematics 87, Springer-Verlag (1994).

[5] R. Brown and C. Spencer, G-groupoids, crossed modules and the fundamental groupoid of a topological group, Nederl. Akad. Wetensch. Proc. Ser. A 79=Indag. Math. 38 (1976), no. 4, 296-302.

[6] J.L. Brylinski, Loop Spaces, Characteristic Classes and Geometric Quantization, Birkhäuser, Boston (1993).

[7] A. D'Agnolo and P. Polesello, Stacks of twisted modules and integral transforms, Geometric aspects of Dwork's theory, Vol. I, II, Walter de Gruyter GmbH \& Co. KG, Berlin (2004), pp. 463-507

[8] S. Eilenberg and S. MacLane, Relations between homology and homotopy groups of spaces, Ann. of Math. (2) 46, (1945), 480-509.

[9] J. Giraud, Cohomologie non abélienne, Grundlheren der Math. Wiss. 179, Springer-Verlag (1971).

[10] K.A. Hardie, K.H Kamps, R.W. Kieboom A homotopy bigroupoid of a topological space Appl. Categ. Structures 9 (2001), no. 3, 311-327.

[11] T. Leinster, Higher operads, higher categories, London Mathematical Society Lecture Note Series, 298. Cambridge University Press, Cambridge, 2004. xiv +433 pp.

[12] S. MacLane, Categories for the working mathematician, Graduate Texts in Math. 5, Springer 2nd ed. (1998).

[13] B. Toen, Vers une interpretation Galoisienne de la theorie de l'homotopie, Cahiers de Top. et Geom. Diff. Cat., Vol. XLIII-4 (2002), 257-312.

[14] I. Waschkies, The stack of microlocal perverse sheaves, Bull. Soc. Math. France 132 (2004), no. 3, 397-462. 
[SGA1] A. Grothendieck, Revêtements étales et groupe fondamental. With contributions by M. Raynaud. Lecture Notes in Mathematics 224, Springer (1971).

[SGA4] M. Artin, A. Grothendieck, and J.L. Verdier, Théorie des topos et cohomologie étale des schémas. Tome 1: Théorie des topos. Lecture Notes in Mathematics 269, Springer (1972).

This article may be accessed via WWW at http://www.rmi.acnet.ge/hha/ or by anonymous ftp at

ftp://ftp.rmi.acnet.ge/pub/hha/volumes/2005/n1a7/v7n1a7.(dvi,ps,pdf)

Pietro Polesello pietro@math.unipd.it

Università di Padova

Dipartimento di Matematica

via G. Belzoni, 735131 Padova,

Italy

Ingo Waschkies ingo@math.unice.fr

Laboratoire J.A. Dieudonné

Université de Nice Sophia-Antipolis

Parc Valrose, 06108 Nice Cedex 2,

France 